\title{
Surveys of fortified sites in Southern Wallacea
}

\author{
Sue 0'Connor, Shimona Kealy, Andrew McWilliam, Sally Brockwell, \\ Lucas Wattimena, Marlon Ririmasse, Mahirta, Alifah, Sandra Pannell, \\ Stuart Hawkins, Mohammad Husni and Daud Tanudirjo
}

The chapters in this volume offer some insight into how widespread the phenomenon of fortification was throughout the Wallacean archipelago. Chapter 10, in particular, presents historical information and photographic records for a number of fortified settlements in Maluku, providing details on the number of houses they contained and the way in which they operated as defensive structures. This chapter presents additional commentary, locational information and photographic records of fortified settlements recorded during the course of general archaeological surveys on Sumba, Timor-Leste, Kisar, Babar and Wetang Islands (Figure 11.1), the nature of which will be explained in more detail below.

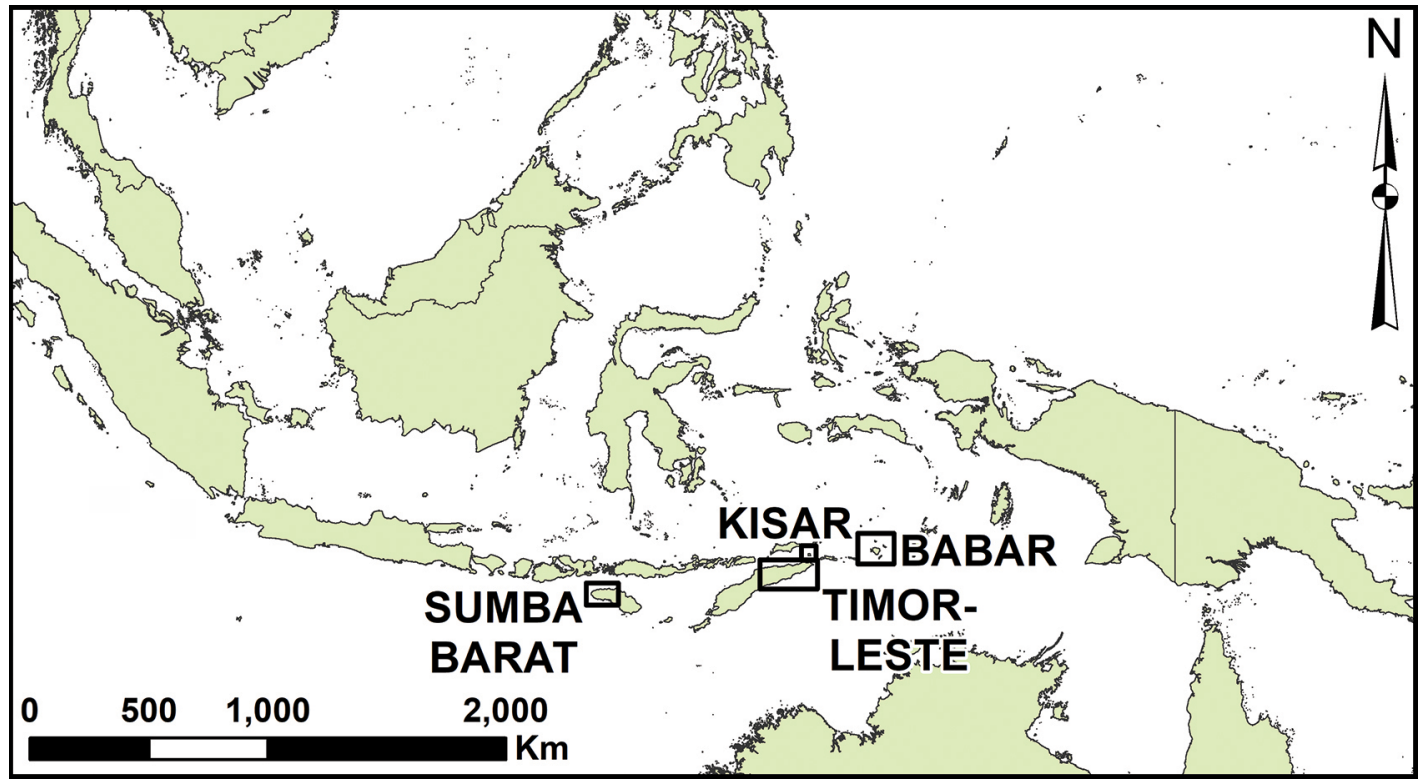

Figure 11.1. Map showing survey locations.

Source: Shimona Kealy, based on coastline data from: 'Global self-consistent, hierarchical, high-resolution geography database'. Version 2.3.7. Released June 2017 (Wessel and Smith 1996). 
Fortified built structures discussed in this chapter have been documented in varying degrees of detail. For some, oral history of settlement use was collected, while for others a GPS location and photographic record was all the information obtained. On some islands, such as small Kisar, pedestrian survey of the uplifted limestone terraces rising from the coast within $1 \mathrm{~km}$ of the coastline was relatively comprehensive, with approximately 70 per cent of the periphery of the island covered. Our survey of the interior of the island, however, was much more ad hoc and areas surveyed were selected on the basis of information provided by local guides. Other areas of Kisar were surveyed using satellite imagery, which was made possible by the low density of vegetation cover. On Babar and Wetang Islands, and on the island of Sumba, the fortified structures described below were found incidentally during the course of fieldwork aimed at locating caves suitable for excavation. Survey of the landscape using satellite imagery failed to reveal fortified sites on these islands due to the dense tree cover and thus lack of ground visibility. On the larger island of Timor, intensive anthropological surveys were undertaken in limited areas, aimed primarily at recording oral histories about land ownership, genealogies, past land use and subsequent resettlement to coastal areas. Collectively these records highlight the remarkable diversity, but also the many shared locational and structural features, of fortified sites in Wallacea.

\section{Fortified settlements in West Sumba}

The western portion of Sumba Island was surveyed in the dry season of 2009 by a joint team from The Australian National University (ANU) and the Universitas Gadjah Mada (Sally Brockwell, Sue O'Connor and Daud Tanudirjo). We were based in the main town of Waikabubuk and took day trips by car to sites accessible by road and on foot. Our survey was not systematic or comprehensive due to the pressure of time. We were told about, or taken to, sites by knowledgeable locals and Dr David Mitchell, an Australian medical doctor who had lived and worked as a volunteer in West Sumba from 1968 to 1975, and who has visited often since then. As well as fortified settlements, we also recorded caves with occupation evidence, old villages, graves and coastal artefact scatters and middens. GPS locations and brief descriptions were recorded but no detailed measurements were taken (Figure 11.2; Table 11.1).

Table 11.1. Summary data on fortified sites in West Sumba.

\begin{tabular}{|l|l|l|l|l|l|}
\hline No. & Kecamatan (subdistrict) & Site name & Location & Description & Findings \\
\hline 1 & Wanukaka & Paletirua & $\begin{array}{l}\text { Lat. }-09.770^{\circ} \\
\text { Long. } 119.405^{\circ} \\
\text { 'Alt. } 147 \mathrm{~m}\end{array}$ & Large fortified village & $\begin{array}{l}\text { Stone walls, megalithic } \\
\text { tombs, laca, pottery }\end{array}$ \\
\hline 2 & Wanukaka & Parimareha & $\begin{array}{l}\text { Lat. }-09.789^{\circ} \\
\text { Long. } 119.389^{\circ} \\
\text { Alt. } 121 \mathrm{~m}\end{array}$ & Fortified village & $\begin{array}{l}\text { 3 houses, high stone } \\
\text { walls }\end{array}$ \\
\hline 3 & Wanukaka & Kulke & $\begin{array}{l}\text { Lat. }-09.792^{\circ} \\
\text { Long. } 119.388^{\circ} \\
\text { Alt. } 124 \mathrm{~m}\end{array}$ & $\begin{array}{l}\text { Fortified village on } \\
\text { top of hill }\end{array}$ & 4 houses, stone walls \\
\hline 4 & Loli & Praigege & $\begin{array}{l}\text { Lat. }-09.554^{\circ} \\
\text { Long. } 119.453^{\circ} \\
\text { Alt. } 539 \mathrm{~m}\end{array}$ & $\begin{array}{l}\text { Old walled village on } \\
\text { hilltop, large: } ~ 200 \mathrm{~m} \\
\text { across }\end{array}$ & $\begin{array}{l}\text { Many internal walls, } \\
\text { broken graves, pottery }\end{array}$ \\
\hline 5 & Loli & Kodarawa Watuoleate & $\begin{array}{l}\text { Lat. }-09.543^{\circ} \\
\text { Long. } 119.489^{\circ} \\
\text { Alt. } 514 \mathrm{~m}\end{array}$ & $\begin{array}{l}\text { Old site on hilltop } \\
\text { above village, small }\end{array}$ & Very rocky. 2 graves \\
\hline 6 & Laratama & Wei Malado & $\begin{array}{l}\text { Lat. }-09.461^{\circ} \\
\text { Long. } 119.414^{\circ} \\
\text { Alt. } 504 \mathrm{~m}\end{array}$ & Fortified village & $\begin{array}{l}\text { Very overgrown, walls, } \\
\text { spirit house, graves }\end{array}$ \\
\hline
\end{tabular}

Note: ${ }^{1}$ Approximate altitude.

Source: Authors' summary. 


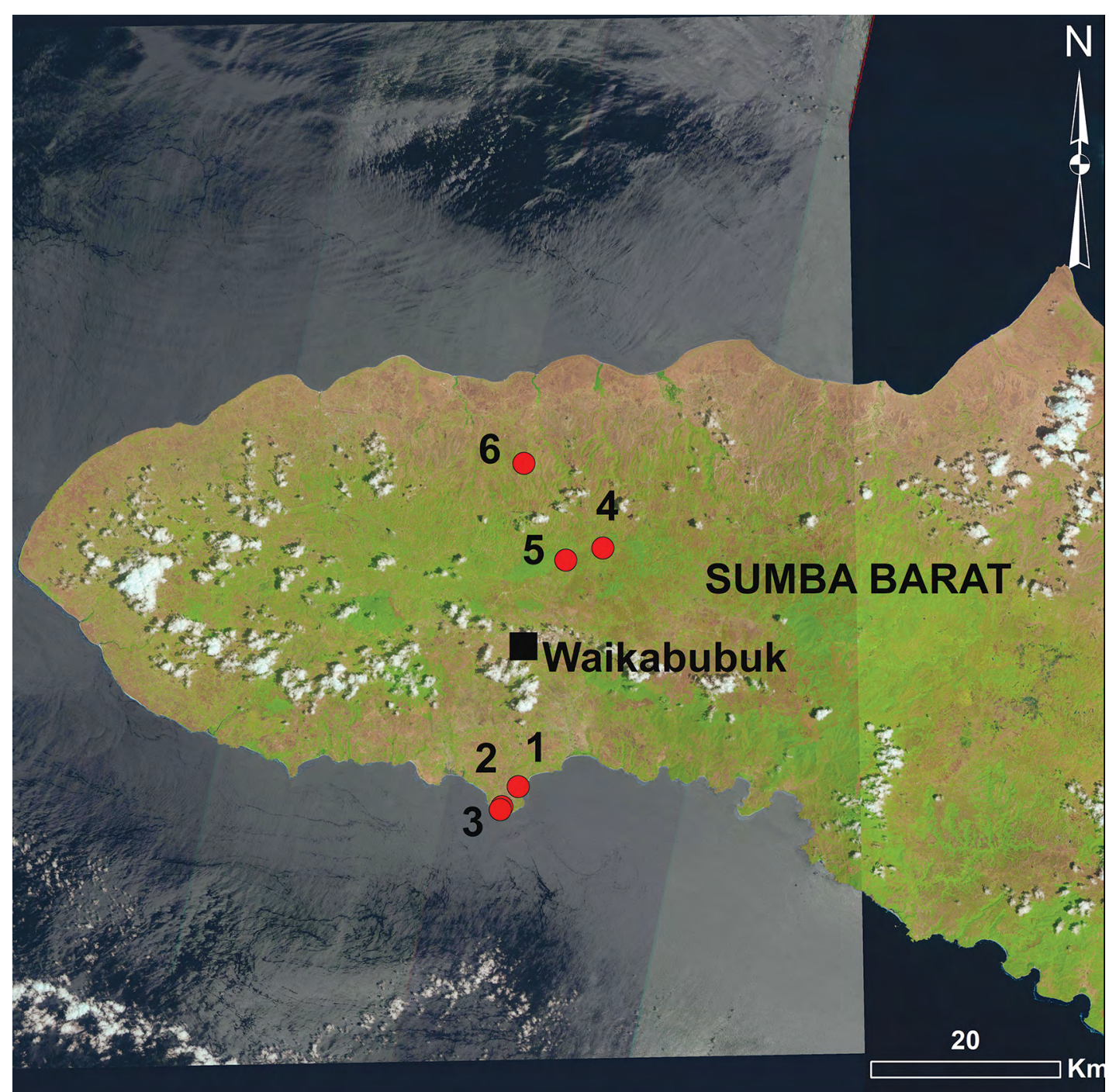

Figure 11.2. Map showing the location of identified fort sites in Sumba Barat (West Sumba).

Sites are numbered based on Table 11.1.

Source: Satellite image from the Sentinel-2A satellite (2019-12-13) obtained from the Sentinel Data Hub (European Space Agency 2019).

The fortified sites described below are all located in defensive positions on hilltops or high cliffs above the sea (Table 11.1; Figure 11.3).

1. Rua is an area with coastal clifftop villages. We visited three villages here, Paletirua, Parimareha and Kulke. Paletirua is a large village with many houses surrounded by stone walls. There is Pandanus growing out of them, which is perhaps a defensive feature. The village is located on a steep cliff, $147 \mathrm{~m}$ above sea level. It contains many megalithic tombstones and a laca-a small open space, which serves as an antechamber prior to accessing the main internal compounds (Figure 11.3). There was pottery by the entrance, including some red slipware.

2. Parimareha is a small village with three houses surrounded by high stone walls. The locals say that it is old.

3. Kulke is a small village with four houses. It is located on top of a hill surrounded by stone walls. At the end of the road next to it, there are steep cliffs rising out of the sea. 
4. Praigege is a village on top of a hill at an elevation of $539 \mathrm{~m}$, overlooking the valley in a southerly direction back towards Waikabubuk. It is surrounded by extensive stone walls that are now damaged. It contains internal stone walls as well. There are many degraded graves, also broken, with a laca in front of the graves. There is much pottery on the surface. The site was very overgrown so visibility was poor, but it appears that it is extensive, about $200 \mathrm{~m}$ across. Our guide told us the village had been abandoned for 100 years.

5. Kodarawa Watuoleate is an old settlement located at an elevation of $514 \mathrm{~m}$ on a rocky hilltop above the present-day village. There used to be walls but the stone has been removed to fence the gardens below. The two graves it contains have been looted; however, they are still regarded as being sacred and are mentioned in adat (customary law and practice) songs.

6. Wei Malado is an abandoned site in the forest at an elevation of c. $500 \mathrm{~m}$. The whole site was overgrown and ground visibility is poor. It contains a spirit house, monumental tomb stones and the remains of a wall with stone slabs. There are as many as 10 clan tombs here.
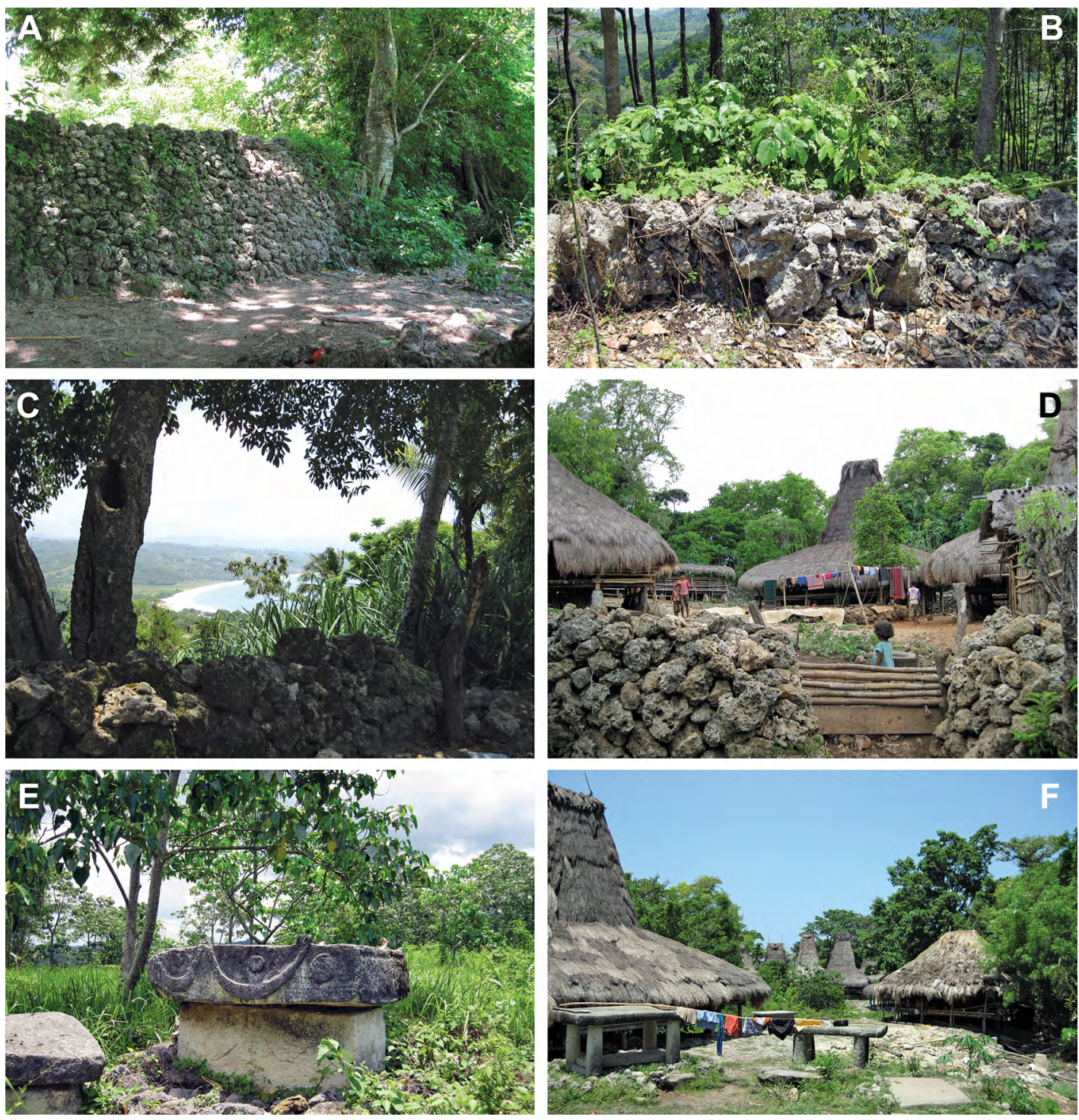

Figure 11.3. Paletirua fortified village.

A. Paletirua high walls; B. Praigege walls; C. Paletirua view to sea; D. Paletirua entrance; E. Praigege tombs; F. Paletirua megalithic tombs.

Source: Photos courtesy of Sally Brockwell. 
David Mitchell recounted an incident that occurred in West Sumba in 1998 (Dibley 1999), which may recall inter-clan conflicts in past times when forts were actively used as defensive positions against raiding parties. The Loli and Wewewa clans are related through marriage, and share in common churches, schools and businesses, despite some disagreements over land boundaries. Although usually peaceful, on 5 November, 2000 Wewewa men, dressed in traditional cloths and headbands and armed with spears, machetes and rocks, marched on Waikabubuk in the Loli Valley. The Wewewa were acting in reprisal against a Loli mob who had attacked them and looted their houses the previous week as the result of an out-of-control demonstration against local government corruption. The raiding Wewewa stormed into Waikabubuk but were met with an opposing Loli force defending Kampung Tarung, the traditional mother village of the Loli district, standing on the hilltop above the modern town, guarding the rice fields below. The Wewewa were routed, 26 people died from machete wounds and many houses were burnt. Police were sent in and peace was restored quickly by the provincial authorities. However, the incident demonstrates the intensity and sensitivity of inter-clan rivalry even into modern times, and emphasises the necessity for defensive strongholds in the past when there was no higher power to impose order.

\section{Fortified settlements in Timor-Leste}

This Timor-Leste compilation brings together the research of a number of contributing authors, completed over a series of exploratory visits to different areas of the territory between 2009 and 2013, and always accompanied by knowledgeable local guides. The listing includes fortified sites identified by local people but not visited by researchers. Table 11.2 presents this data in summary and includes general location information and brief commentary on significance and site features.

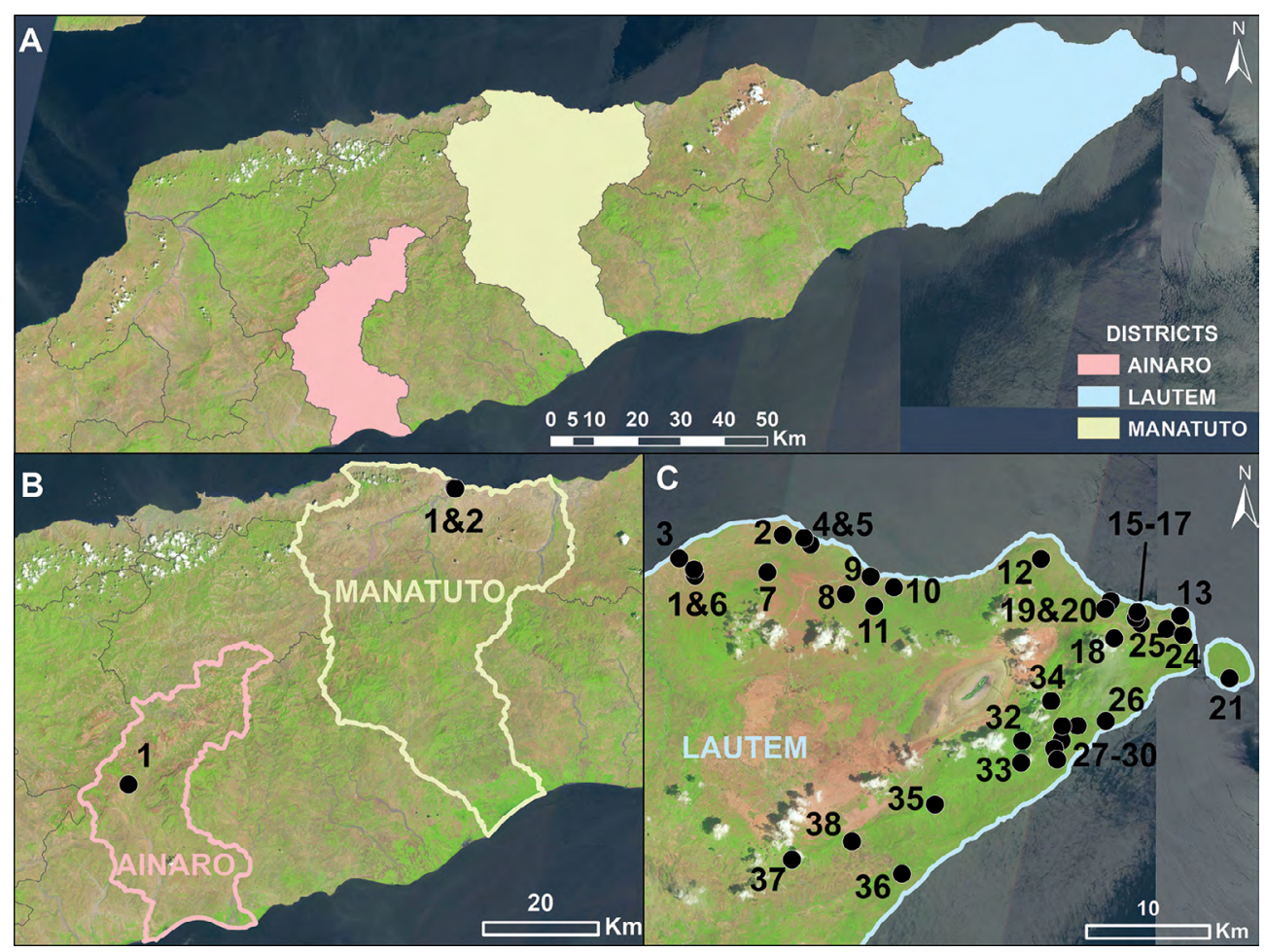

Figure 11.4. Map of fortified sites located in Timor-Leste.

A. Location of the three districts discussed here; B. Sites identified in Ainaro and Manatuto Districts-numbers corresponding to Table 11.2 ; C. Sites identified in Lautem District-numbers corresponding to Table 11.2.

Source: Satellite image from the Sentinel-2A satellite (2019-12-07) obtained from the Sentinel Data Hub (European Space Agency 2019). 


\section{Ainaro District}

The Subago fortified settlement in Ainaro was recorded in May 2010 by Sue O'Connor, Sally Brockwell, and Sandra Pannell. Our local guide, Mateus Anaral, said that there used to be houses built inside the walls and that his grandparents were born there. The remnants of large thick walls are still visible on the southern side of Subago (Figure 11.5A) where there was a single narrow entrance. Several stone 'altars' were observed (Figure 11.5B), including one used during the corn harvest to ensure that the crop is abundant.
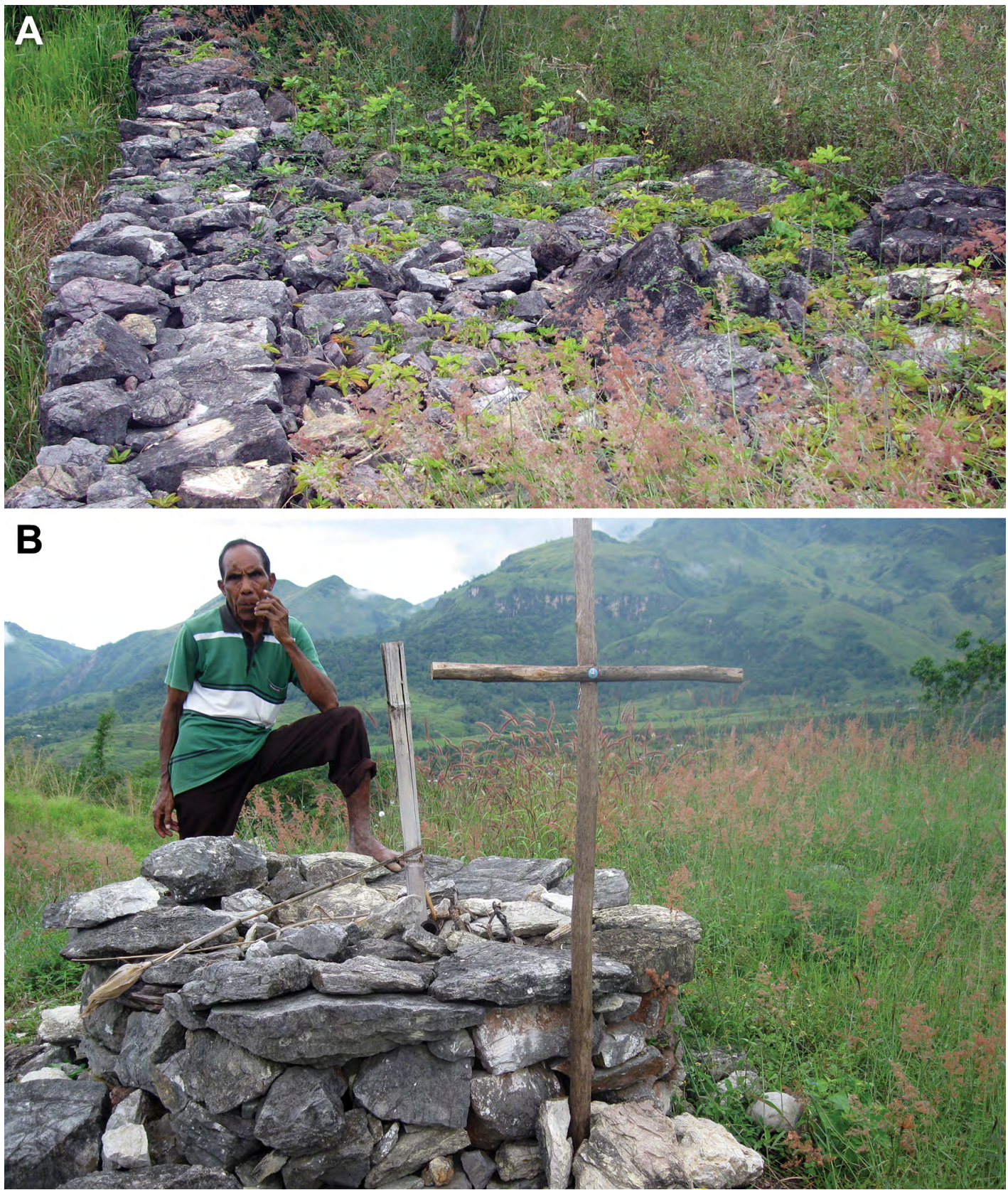

Figure 11.5. Ainaro survey at Subago.

A. Remnant wall; B. Altar.

Source: Photos courtesy of Sue o'Connor. 


\section{Manatuto District}

In 2010, two poorly preserved fortified settlements were recorded within the boundaries of the modern township of Manatuto (Figure 11.4C). The survey was carried out by Sally Brockwell, Sandra Pannell, Sue O'Connor, Estefan Guterres and Guterres Karlilo. Other fortified sites were located on the road between the townships of Manatuto and Laclo but these were not explored in any detail as they had been previously recorded by Chou (2013).

Sau Huhun (Galoli) or Sau Tutun (Tetun) (Chou 2013 provides the name Saututo for this fort) is located on the highest point of the ridgeline overlooking the Manatuto Pousada (rest house) (Figure 11.6). The walls of Sau Huhun could scarcely be made out in places, and we were told that the stone had been removed to build the pousada. Remnant walls occurred around the east, north and west perimeters. The east and west walls could be traced by a low line of rocks. The walls on the west side were a mixture of built-up soil and rock. Inside the walls in the area where we presume the houses would have been constructed, we located Chinese tradeware, earthenware pottery and marine shells (Figure 11.7).
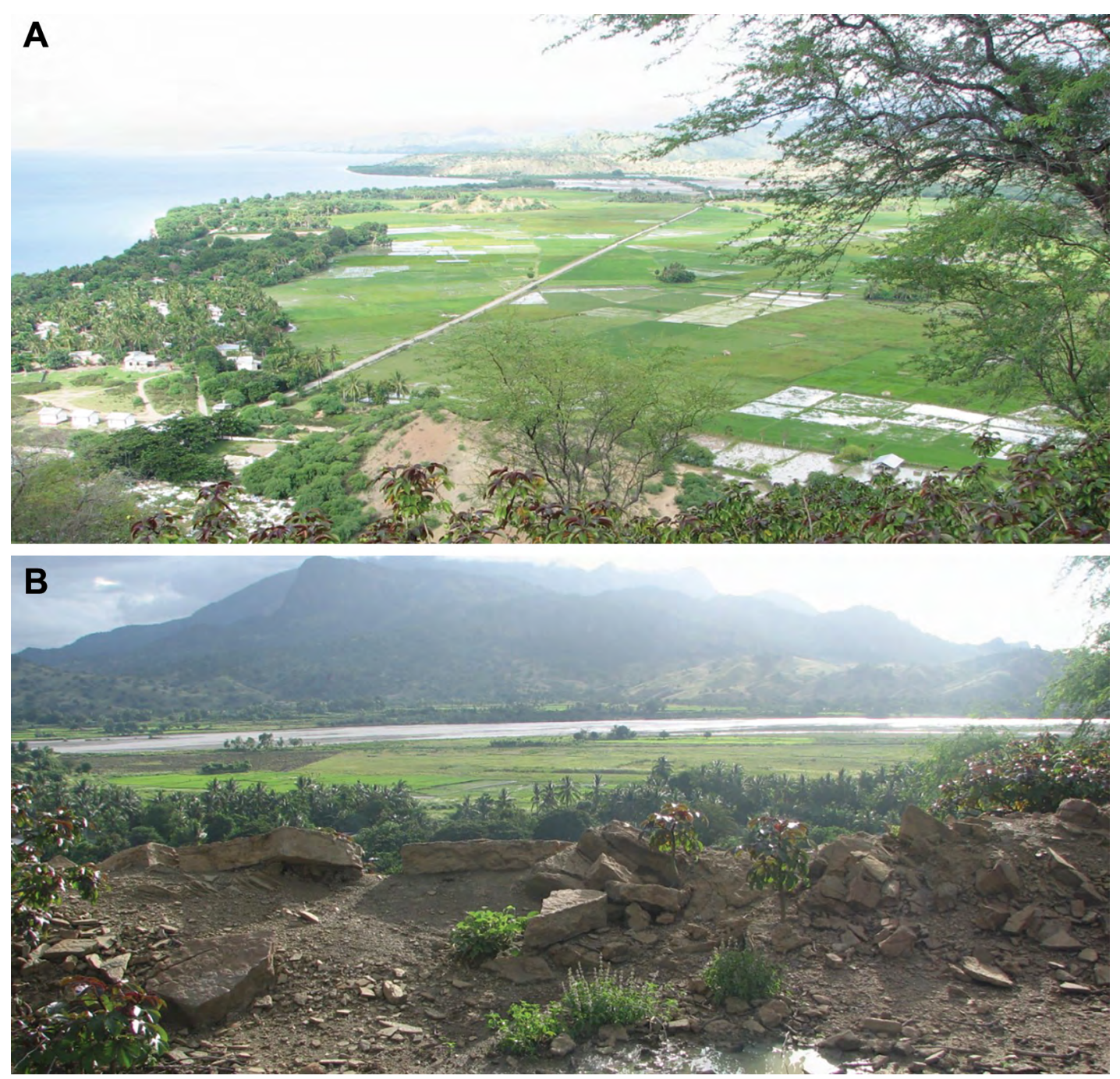

Figure 11.6. Views towards Manatuto from the remains of the Sau Huhun fortification, showing its defensive position.

Source: Photos courtesy of Sue $0^{\prime}$ Connor. 

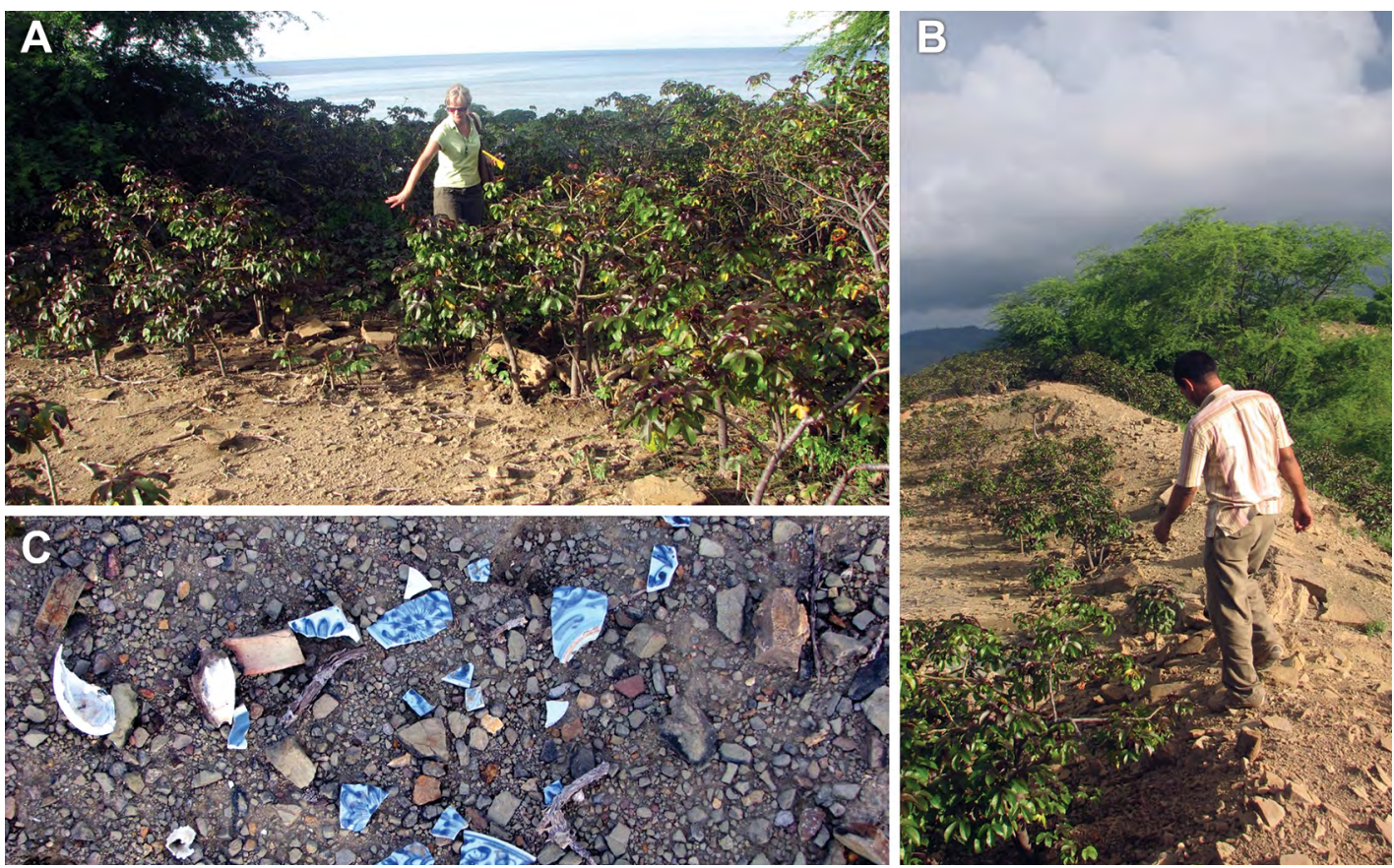

Figure 11.7. Sau Huhun fortification.

A. Sally Brockwell pointing to remnant of wall at northern end of the fort; B. Estefan Guterres walking along earth and rock wall remnant; C. Earthenware sherds, broken Chinese Blue and White tradeware and marine shellfish on the surface at Sau Huhun.

Source: Photos courtesy of Sue o'connor.

On the hilltop opposite the Banda Maria shrine is another fortified settlement with remnant walls. The hill is called Iliheu Tatua (Galoli, $i l i=$ village, heu $=$ new, and tatua $=$ old) . According to our guide Guterres Karlilo, the hilltop was the former site of a village that was later moved to the edge of the river Meta Laclo during the time of the Indonesian occupation from 1975 to 1999 . Remnants of the base of a thick wall on the south side indicate that it was built using two roughly parallel rows of stone infilled with earth. At the base of this hill is a place where there was previously an altar with skulls (Figure 11.8; see Chapter 1, this volume) and in the past fragments of human cranium could still be seen near a large flat stone at this location (Guterres Karillo pers. comm.). They have now disappeared, either taken by people or decayed.

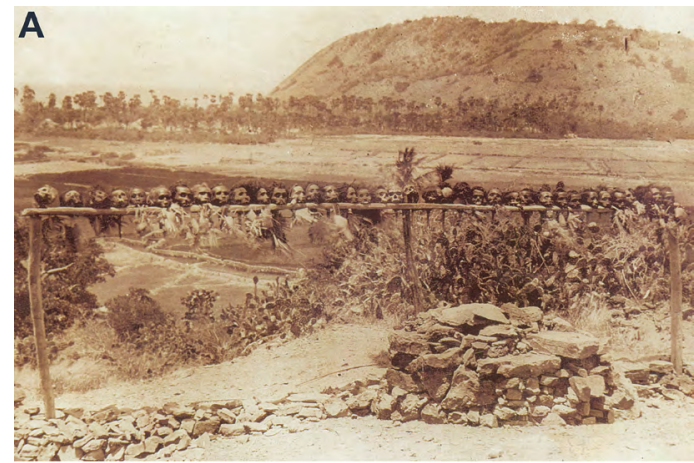

B

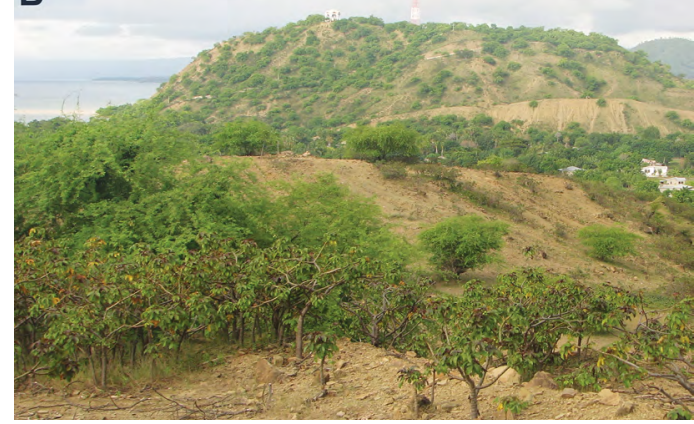

Figure 11.8. Iliheu Tatua hill.

A. Photograph taken in 1913 showing altar with skulls; B. The same view in 2010. The skulls and altar are no longer present. Source: Photo A by António Nascimento Leitão, courtesy of Natural History and Science Museum of University of Porto, register number MHNC-UP-FCUP-IA-AF-775; Photo B courtesy of Sue $0^{\prime}$ Connor. 


\section{Lautem District}

The data in Table 11.2 only reveals a partial view and for the most part focuses on five administrative villages in Lautem municipality (Figure 11.4D). They include Moro-Parlamento, Com, Mehara, Tutuala and Muapitine. A full survey of Lautem itself would likely produce several times this number. Analysis of the named forts, Macapainara (Ili Vali) and Vasino (Moro-Parlamento) are given fuller treatment in Chapters 2 and 4 of this volume and are not addressed here in detail.

During June 2009 surveys were undertaken of the Tutuala area including the Vero River valley and associated forested lands on the seaward side of the Paichao mountain range. ${ }^{1}$ The purpose was to locate and undertake visits to a sample of the fortified settlement sites in the region and begin a process of documenting the range and variation found amongst them.

A large number of fortified sites are located in Tutuala subdistrict (see Lape 2006). One of these is the massive fortification Mapulu, located on a forested hilltop in the upper reaches of the Vero River valley, to the south of the current settlements of Cailoro and Vero (Tutuala subdistrict) (Figure 11.4D). The northeastern side of the fort is strategically located on a limestone cliff with a massive dry stone wall providing a secure inner perimeter. A series of low outlying ring walls extend outward on the lower slopes, with large standing stones providing defensive posts. Access to the fort is via a sloping path lined by $4 \mathrm{~m}$ high stone walls, including apertures that allow defenders to observe and fire upon intruders, probably with Portuguese-style matchlock muskets or local copies that were in general use from the sixteenth century (Lieberman 2009:422). They were equipped with a trigger mechanism to ignite a gunpowder charge that fired a projectile. The muzzle-loading muskets were unwieldy but lethal when on target, and keenly sought along with gunpowder as a trade item of choice in the eastern islands. The gateway to the fort features a laca. Within the fort, the internal space is divided into two general living areas that contain a series of old stone graves (calu luturu) and separate open ritual spaces (sepu) (Figure 11.9).

According to local knowledge, the full name of the fort is Mapulu Ro Malae. The phrase references a number of groups who resided there and continue to maintain relations of sacrificial veneration to the ancestral presence in the site. This occurs at least annually on the Catholic holy day of All Souls' Day (2 November), known as vaci i huma'ara, when relatives visit to clean the graves and ritual spaces and present offerings of candles, betel nut and food to the ancestors. Renu Ratu is the customary land owner (mua ocawa, landlord) and responsible for overall site protection. Another group of customary owners of the site is the clan Kukulori, of which the former Falintil guerrilla resistance leader, Konis Santana, was an integral member who found refuge in the site during the armed struggle for independence. Local custodians of the site recall one historical occasion when they fought off the attack of a Portuguese figure, Kiri Kiri (Gregorio) Maulaka, who had marched from Lorehe and attacked the fort with a large contingent of soldiers. They held large boulders above the access path and when the enemy approached the fort they cut the ropes and crushed many of the soldiers. The internal space of Mapulu contains a ritual altar stone (tei), that represents the spirit guardian of the land to which offerings and invocations are directed when assistance is sought by the living group of members.

1 The Vero valley survey was undertaken Sue O’Connor, Andrew McWilliam, local guides Mario Dos Santos Loiola, Joao Dos Santos and Martinho Dos Santos, and state forestry officers. 

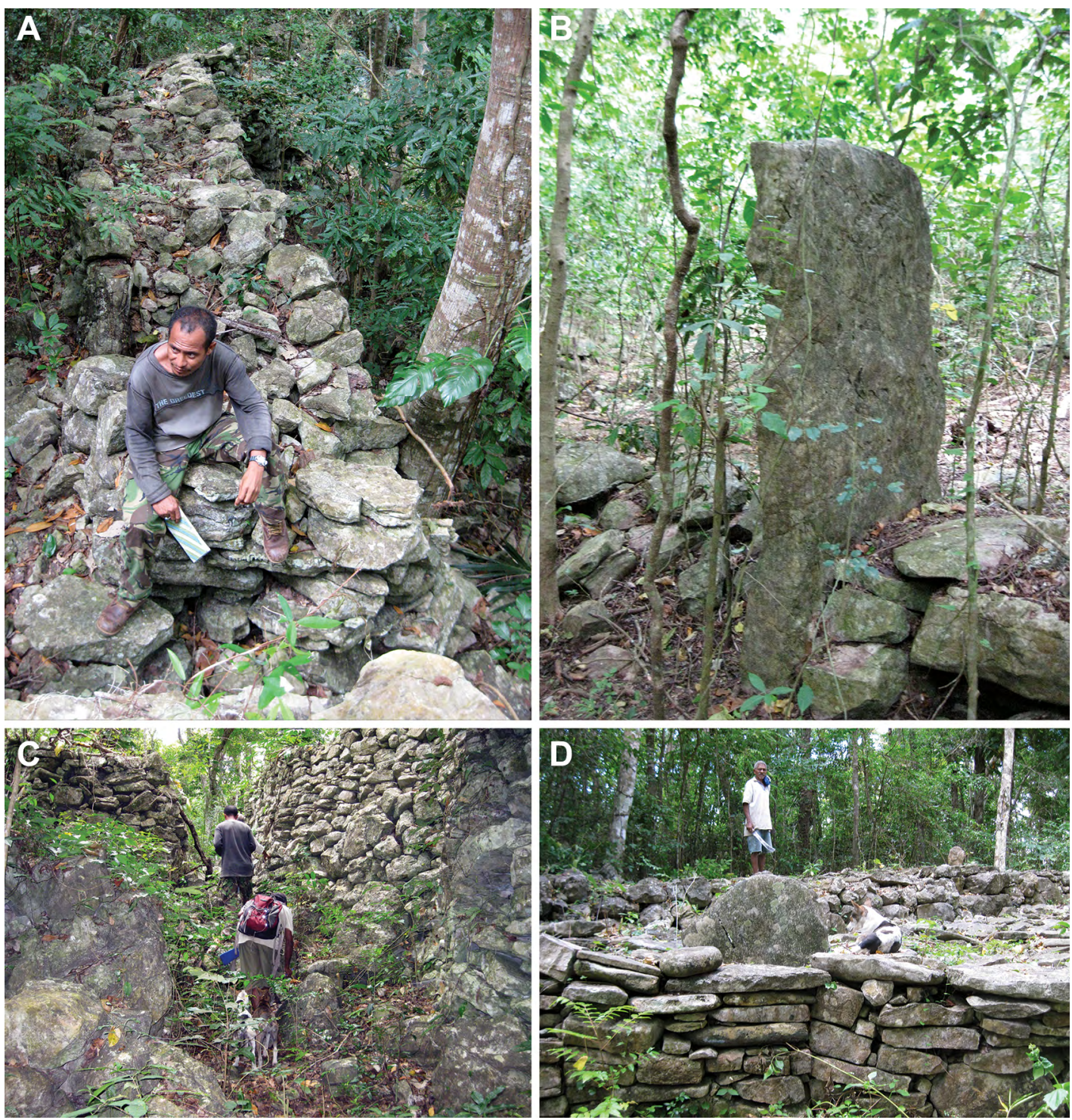

Figure 11.9. Mapulu Ro Malae.

A. Perimeter wall; B. Standing stone; C. Entrance to fort; D. Ancestral graves and sitting area.

Source: Photos courtesy of Andrew McWilliam.

A second example of fortifications in the Tutuala area is the hilltop fort Hi Maka Loli, which is located to the northeast of Mapulu fort and immediately south of the main administrative centre of Tutuala. The fortified site has remnant stone walls and a damaged stone gateway that leads to a double internal space. There are numerous large stone graves with pre-Christian headstones ( $n$ a otu) to which sacrificial offerings are made. The ground has extensive scatters of porcelain and tradeware and includes a ritual dancing area with elevated sitting stones (graves) around the space.

According to local knowledge, the fort (pa'amakolo) is the historical site for the Vacumura paternal origin group, who moved here following the arrival of their ancestors in Timor-Leste. They maintained trade relations with external visitors and recall using João beach on the north coast of Tutuala as a site where trading and exchange occurred. Over time, as the Vacumura group flourished and expanded, the area became too crowded (matete, narrow, crowded). The group largely dispersed to the west, towards the 'wide lands' (mua maluere) of Los Palos. 


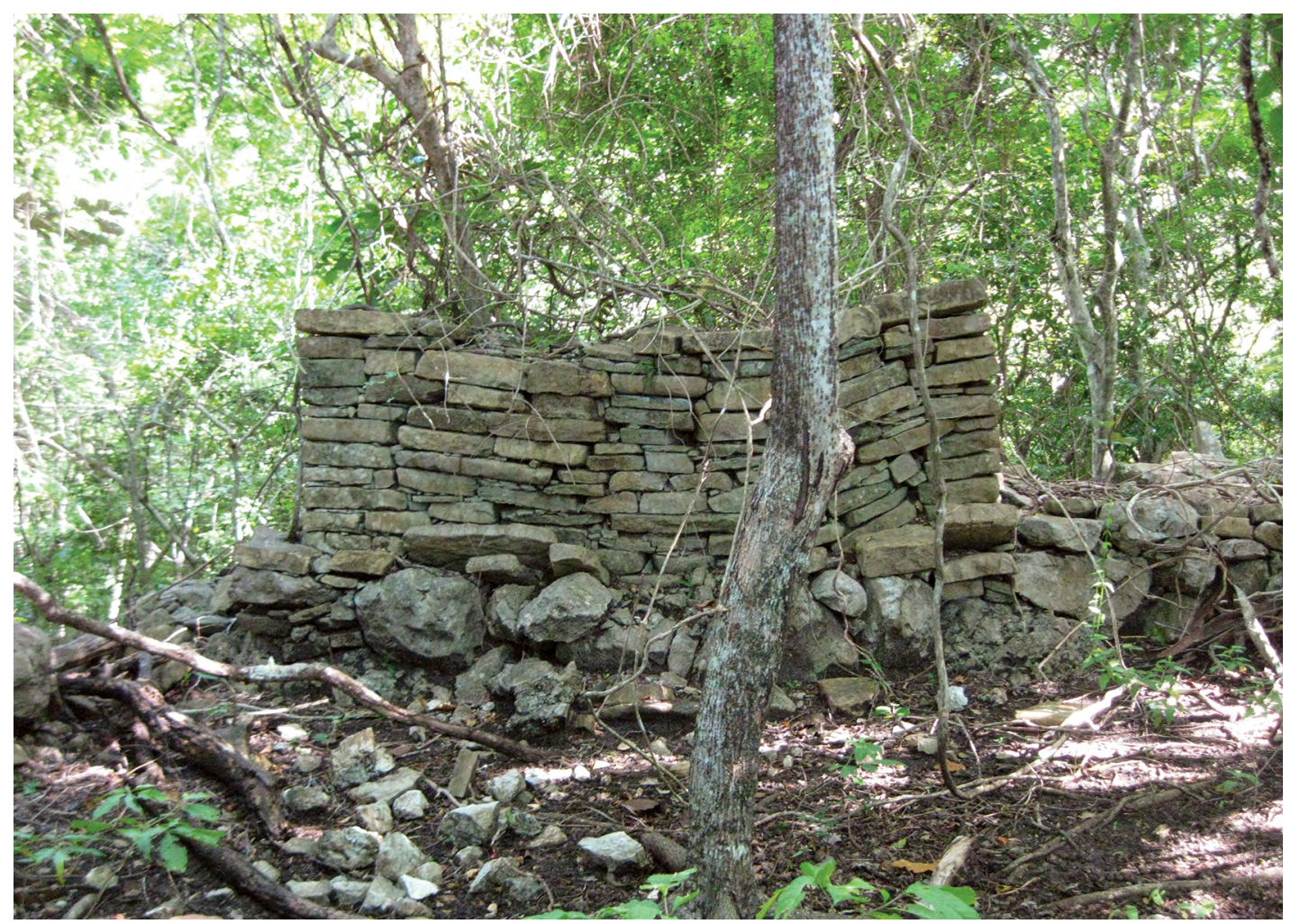

Figure 11.10. Maiana tomb.

Source: Photo courtesy of Andrew McWilliam.

Access to the Vero River valley area is possible via a well-trodden footpath that leads from the settlement of Vero (Tutuala) past the forested hilltop fort of Mapulu, through a series of swidden gardens. It then follows the slope of the Vero River course towards the coast. On the lower reaches of the river, our group visited a range of ancestral locations, including a series of former fortified residential sites that are located at prominent elevated locations on the forested slopes between the Pai Chao mountains and the southeast coast (for extended discussion see Fitzpatrick et al. 2012:160-164).

Fortified sites visited in the Vero valley include Haka Paku Leki, Ili Haraku, Maiana and Pailopo (see Table 11.2 below), all of which were occupied until the early twentieth century but remain important places for ancestral veneration. Each of these sites reflects structural accommodation to the physical properties of the sites, which were often built on existing rocky outcrops and atop cliff faces. Ili Haraki, near the base of the Pua Loki mountain, for example, is fashioned out of a massive rockpile with tactically placed dry stone walls to effect a secure perimeter.

Maiana is one of the smaller fortified sites we recorded. It is situated on a forested hilltop and surrounded by a broadly circular stone wall of substantial proportions. The defensive perimeter also includes areas of spiky cactus (prickly pear) that served as another form of barrier against attack in the past. Maiana is located in the western part of the Vero region close to the historical boundary (the river, Verkass ver) that separated the lands of Fataluku clan groups Renu Ratu and Latuloho Ratu based in the adjacent village of Muapitine. The fort site itself is associated with the clan group Aca Cao, which took over from a former group, Pai' ir Ratu, which died out. The site features a large $(3 \times 1.5 \times 1 \mathrm{~m})$ rectangular grave decorated with dressed stone in the style known as 'Makassar mataru'-Makassar stone (Figure 11.10; also see McWilliam et al. 2012). It sits adjacent to a sepu ritual ground in the usual style of the region. There is no local record of previous fighting at the site. 


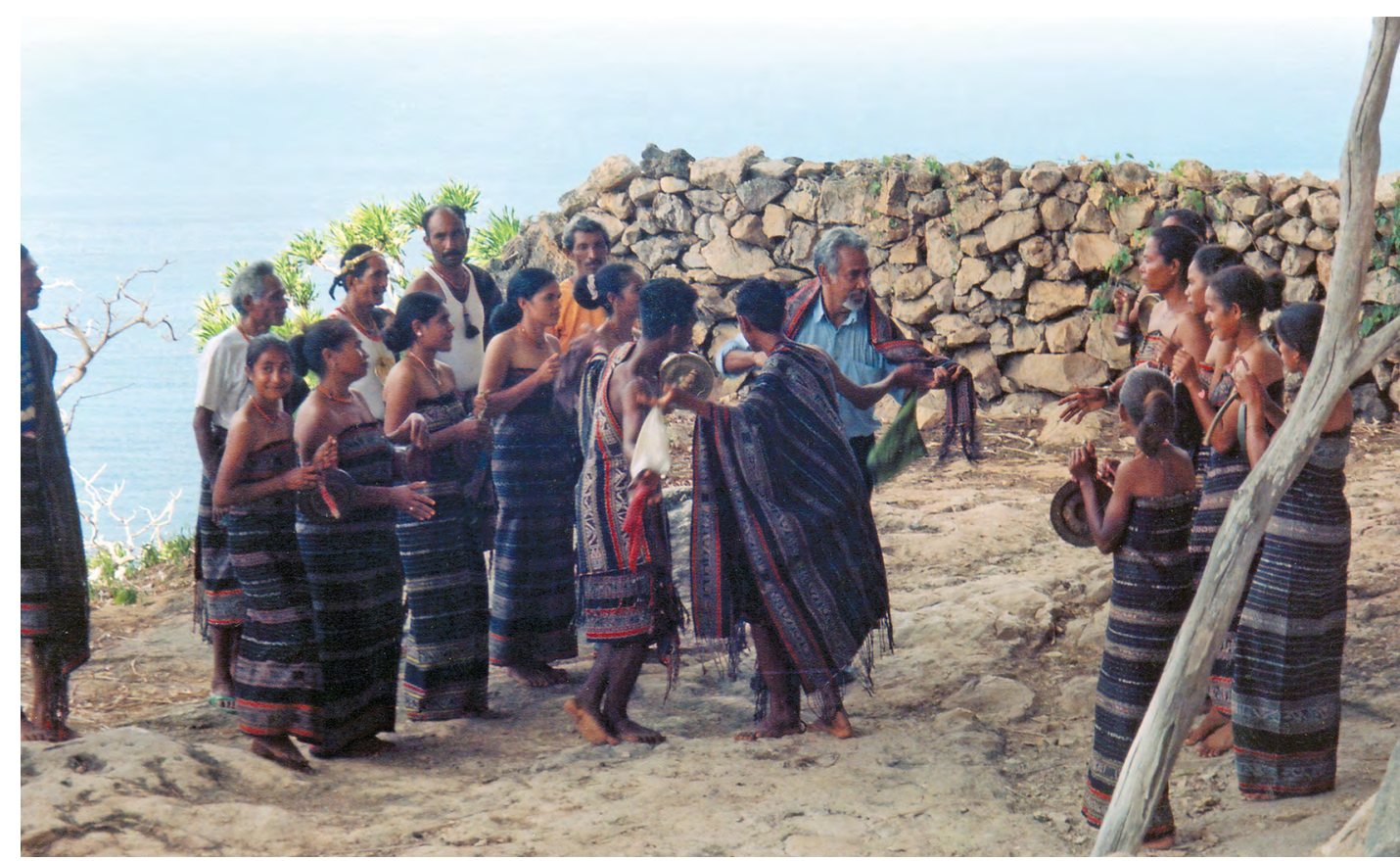

Figure 11.11. Ili Kere Kere, a small overhang on a sheer cliff face in the Tutuala area with stone wall and dancers.

Source: Photo courtesy of Andrew McWilliam. See also Table 11.2 \#13.

Table 11.2. Summary data on fortified sites in Timor-Leste, numbered by district and corresponding to Figure 11.4 .

\begin{tabular}{|c|c|c|c|c|c|}
\hline No. & Subdistrict & Site name & Location & Description & Notes \& significance \\
\hline \multicolumn{6}{|c|}{ AINARO DISTRICT } \\
\hline 1 & Ainaro & Subago & $\begin{array}{l}\text { Lat. }-08.97743^{\circ} \\
\text { Long. } 125.50146^{\circ} \\
\text { Alt. } 1059 \mathrm{~m} \\
\text { On hilltop next to Ainaro } \\
\text { town }\end{array}$ & $\begin{array}{l}\text { Fortified village } \\
\text { with large wall on } \\
\text { the south side and } \\
\text { a single exit and } \\
\text { entrance. }\end{array}$ & $\begin{array}{l}\text { Has several stone altars which } \\
\text { include one used during corn } \\
\text { harvest. }\end{array}$ \\
\hline \multicolumn{6}{|c|}{ MANATUTO DISTRICT } \\
\hline 1 & Manatuto & Sau Huhun & $\begin{array}{l}\text { Lat. }-08.30936^{\circ} \\
\text { Long. } 126.00586^{\circ} \\
\text { Alt. } 72 \mathrm{~m} \\
\text { On top of hill, opposite } \\
\text { and looking to Manatuto } \\
\text { Pousada. }\end{array}$ & $\begin{array}{l}\text { Old Settlement; Has } \\
\text { walls on } \mathrm{E}, \mathrm{W} \text { and } \\
\mathrm{N} \text { sides but only } \\
\text { low line of rocks } \\
\text { remaining. }\end{array}$ & $\begin{array}{l}\text { Chinese tradeware, pottery, marine } \\
\text { shellfish on surface. }\end{array}$ \\
\hline 2 & Manatuto & $\begin{array}{l}\text { lliheu Tatua } \\
\text { (name of } \\
\text { hill) }\end{array}$ & $\begin{array}{l}\text { Lat. }-08.51457^{\circ} \\
\text { Long. } 126.01263^{\circ} \\
\text { Hilltop opposite Bunda } \\
\text { Maria shrine. }\end{array}$ & $\begin{array}{l}\text { Old settlement with } \\
\text { altar, remnants of } \\
\text { wall on S side. }\end{array}$ & $\begin{array}{l}\text { At the base of the hill skulls } \\
\text { are placed above a stone altar; } \\
\text { marine shellfish and pottery seen } \\
\text { on surface. }\end{array}$ \\
\hline \multicolumn{6}{|c|}{ LAUTÉM DISTRICT } \\
\hline 1 & $\begin{array}{l}\text { Moro- } \\
\text { Parlamento }\end{array}$ & $\begin{array}{l}\text { Vasino or } \\
\text { Wasino }\end{array}$ & $\begin{array}{l}\text { Lat. }-08.36475^{\circ} \\
\text { Long. } 126.93531^{\circ} \\
\text { Alt. } 245 \mathrm{~m} \\
\text { On the southern edge } \\
\text { of Moro settlement in } \\
\text { the hill above the north } \\
\text { coast (see Chapter 4, } \\
\text { this volume). }\end{array}$ & $\begin{array}{l}\text { Site has a large stone } \\
\text { grave that is reported } \\
\text { to be a group burial } \\
\text { tomb (Poku caru, to } \\
\text { open the basket). } \\
\text { Used in living memory } \\
\text { but no longer due to } \\
\text { Church disapproval. }\end{array}$ & $\begin{array}{l}\text { Former major centre for Pai'ir } \\
\text { (Moro) Ratu (also known as Kota } \\
\text { lulunu). Fort built as result of } \\
\text { outbreak of warfare following arrival } \\
\text { of Portuguese. Authority granted } \\
\text { to related rival group in Lautem. } \\
\text { (Uruha'a Ratu-Dom Paulo). José le } \\
\text { Sumalai was last 'king' of Vasino } \\
\text { before people moved. }\end{array}$ \\
\hline
\end{tabular}




\begin{tabular}{|c|c|c|c|c|c|}
\hline No. & Subdistrict & Site name & Location & Description & Notes \& significance \\
\hline 2 & $\begin{array}{l}\text { Moro- } \\
\text { Parlamento }\end{array}$ & Vasaku & $\begin{array}{l}\text { Lat. }-08.33500^{\circ} \\
\text { Long. } 126.99935^{\circ} \\
\text { Alt. } 115 \mathrm{~m}\end{array}$ & $\begin{array}{l}\text { Parma kolo. Has } \\
\text { intact walls up to } \\
1.5 \mathrm{~m} \text {. Only one wall } \\
\text { remains. Has double } \\
\text { grave. }\end{array}$ & \\
\hline 3 & $\begin{array}{l}\text { Moro- } \\
\text { Parlamento }\end{array}$ & $\begin{array}{l}\text { Laulau } \\
\text { Lokotu }\end{array}$ & $\begin{array}{l}\text { Lat. }-08.3520^{\circ} \\
\text { Long. } 126.9237^{\circ} \\
\text { Alt. } 40 \mathrm{~m} \\
\text { On the eastern side } \\
\text { of the road to Moro } \\
\text { from coast. }\end{array}$ & $\begin{array}{l}\text { A semi-fortified site } \\
\text { overlooking rice fields } \\
\text { on the coast. }\end{array}$ & $\begin{array}{l}\text { Said to be dwelling place before } \\
\text { relocation to Vasino. Original name } \\
\text { of Moro is Pai Sapolo. Most stone } \\
\text { repurposed to make road to Moro } \\
\text { during Portuguese times. }\end{array}$ \\
\hline 4 & $\begin{array}{l}\text { Moro- } \\
\text { Parlamento }\end{array}$ & Masui & $\begin{array}{l}\text { Lat. }-08.33731^{\circ} \\
\text { Long. } 127.01489^{\circ} \\
\text { Alt. } 85 \mathrm{~m} \\
\text { Beside road west of the } \\
\text { settlement of Ira Ara } \\
\text { (west of Com). }\end{array}$ & $\begin{array}{l}\text { Fort has been partly } \\
\text { dismantled with } \\
\text { remnant walls and } \\
\text { entrance stones } \\
\text { standing (see } \\
\text { 0'connor et al. 2012). }\end{array}$ & $\begin{array}{l}\text { Kati Ratu owner of area but fort } \\
\text { long abandoned. } \\
\text { Site has prominent wooden } \\
\text { structure-said to be burial (see } \\
\text { Figure } 1.4 \text { ). Graves with } 2 \text { uprights. } \\
\text { Fort said to have been built by } \\
\text { magical means (masino). }\end{array}$ \\
\hline 5 & $\begin{array}{l}\text { Moro- } \\
\text { Parlamento }\end{array}$ & Іга Ага & $\begin{array}{l}\text { Lat. }-08.341797^{\circ} \\
\text { Long. } 127.019384^{\circ} \\
\text { Alt. } 100 \mathrm{~m} \\
\text { Near Ira Ara village, } \\
\text { adjacent to large spring } \\
\text { and main road to Com. }\end{array}$ & $\begin{array}{l}\text { See Lape et al. } \\
\text { (Chapter } 3 \text {, this } \\
\text { volume). }\end{array}$ & $\begin{array}{l}\text { Old site formerly built by Cailoro } \\
\text { Ratu who married daughter of } \\
\text { Kati Ratu in a classic example of a } \\
\text { stranger king mythology (Sahlins } \\
\text { 1985:87). }\end{array}$ \\
\hline 6 & $\begin{array}{l}\text { Moro- } \\
\text { Parlamento }\end{array}$ & Ira Сао & $\begin{array}{l}\text { Lat. }-08.36022^{\circ} \\
\text { Long. } 126.93450^{\circ} \\
\text { Alt. } 167 \mathrm{~m} \\
\text { Directly above church in } \\
\text { modern village of Moro, } \\
\text { SE of and above the } \\
\text { freshwater spring. }\end{array}$ & $\begin{array}{l}\text { Low walls less than } \\
1 \mathrm{~m} \text { high, as stone } \\
\text { has been reused } \\
\text { for buildings in } \\
\text { modern village. Stone } \\
\text { walls and graves } \\
\text { overgrown. } \\
\end{array}$ & $\begin{array}{l}\text { Old fortified settlement. } \\
\text { chronological period unknown } \\
\text { but used before and into } \\
\text { Portuguese times. }\end{array}$ \\
\hline 7 & Moro-Nari агеа & $\begin{array}{l}\text { Ili Fanu, } \\
\text { Apa Fanu }\end{array}$ & $\begin{array}{l}\text { On high slopes to the } \\
\text { south of Soekili village, } \\
\text { Nari. }\end{array}$ & $\begin{array}{l}\text { Fortified site. Not } \\
\text { visited. }\end{array}$ & $\begin{array}{l}\text { Little information-Kati Ratu likely } \\
\text { (see Viegas and Feijó 2019). }\end{array}$ \\
\hline 8 & Com агеа & Lor Lafae & $\begin{array}{l}\text { Overlooking the port } \\
\text { of Com to the east of } \\
\text { Etepiti settlement, } 1 \mathrm{~km} \\
\text { inland. }\end{array}$ & $\begin{array}{l}\text { Strategic fortified } \\
\text { site. Extensive } \\
\text { area of limestone } \\
\text { cliffs and walled } \\
\text { areas. A number of } \\
\text { significant graves. }\end{array}$ & $\begin{array}{l}\text { Fortified settlement of Konu } \\
\text { Ratu-main group of Com, } \\
\text { which historically controlled } \\
\text { trading at the port/anchorage } \\
\text { (McWilliam 2007a). }\end{array}$ \\
\hline 9 & Com агеа & O'o Lo Kon & $\begin{array}{l}\text { Lat. }-08.3652^{\circ} \\
\text { Long. } 127.0633^{\circ} \\
\text { Alt. } 65 \mathrm{~m} \\
\text { Immediately above the } \\
\text { beach and overlooking } \\
\text { the harbour of Com. }\end{array}$ & $\begin{array}{l}\text { Walled site with } \\
\text { numerous stone } \\
\text { graves. }\end{array}$ & $\begin{array}{l}\text { Early fortified site for Konu Ratu } \\
\text { who historically controlled trade in } \\
\text { the port (see McWilliam 2007a). }\end{array}$ \\
\hline 10 & Com агеа & Ili Vali & $\begin{array}{l}\text { Lat. }-08.37344^{\circ} \\
\text { Long. } 127.08045^{\circ} \\
\text { Alt. } 200 \mathrm{~m} \\
\text { On bluff overlooking } \\
\text { coast } 3 \mathrm{~km} \text { east of Com. }\end{array}$ & $\begin{array}{l}\text { Walled site with } \\
\text { extensive internal } \\
\text { areas of graves, } \\
\text { dancing ground } \\
\text { and other features. } \\
\text { Developed on two } \\
\text { levels (see Chapter 2, } \\
\text { this volume). } \\
\end{array}$ & $\begin{array}{l}\text { Main fortified settlement of } \\
\text { Fara kati Ratu (Macapainara- } \\
\text { Serevairara). Appointed Koronel } \\
\text { by Portuguese (18th C.). Moved } \\
\text { to near coast became Mua Pusu } \\
\text { village. In } 1976 \text { forcibly relocated } \\
\text { to Com by Indonesian military. }\end{array}$ \\
\hline 11 & Com агеа & $\begin{array}{l}\text { Loho Matu } \\
\text { Lata }\end{array}$ & $\begin{array}{l}\text { About } 2 \mathrm{~km} \text { above the } \\
\text { coast and Mua Pusu } \\
\text { settlement area. East } \\
\text { of Com. }\end{array}$ & $\begin{array}{l}\text { Strategic fortified } \\
\text { location overlooking } \\
\text { forested slopes to the } \\
\text { coast below. }\end{array}$ & $\begin{array}{l}\text { Large number of old graves, tei } \\
\text { altars and crumbling stone walls. } \\
\text { Actively managed by Loho Matu } \\
\text { community in Com. }\end{array}$ \\
\hline
\end{tabular}




\begin{tabular}{|c|c|c|c|c|c|}
\hline No. & Subdistrict & Site name & Location & Description & Notes \& significance \\
\hline 12 & Luikere area & $\mathrm{N} / \mathrm{A}$ & $\begin{array}{l}\text { North of Mehara } \\
\text { settlement } \\
\text { (east of } \mathrm{Com} \text { ). }\end{array}$ & $\begin{array}{l}\text { Fortified settlement. } \\
\text { Not visited. }\end{array}$ & Former stronghold of Kapitan Ratu. \\
\hline 13 & $\begin{array}{l}\text { Tutuala-Mehara; } \\
\text { northern area }\end{array}$ & $\begin{array}{l}\text { Ili Kere } \\
\text { Kere }\end{array}$ & $\begin{array}{l}\text { Lat. }-08.393911^{\circ} \\
\text { Long. } 127.289482^{\circ} \\
\text { Alt. } 250 \mathrm{~m} \\
\text { Clifftop fortified cave } \\
\text { site. }\end{array}$ & $\begin{array}{l}\text { Series of overhangs } \\
\text { with sheer cliffs } \\
\text { on eastern side, } \\
\text { partitioned by } \\
\text { stone walls } \\
\text { (see Figure } 11.11 \text { ) } \\
\text { with rock art and } \\
\text { sacrificial altar. }\end{array}$ & $\begin{array}{l}\text { Mythic settlement site of Tutuala } \\
\text { Ratu. Origin settlement that had } \\
\text { mythic large wax candle that } \\
\text { attracted seafaring settlers to } \\
\text { Timor. Massive stone walls used } \\
\text { for defence partition space into } \\
\text { three sections (see O'Connor et al. } \\
\text { 2011:49-52 for additional detail). }\end{array}$ \\
\hline 14 & $\begin{array}{l}\text { Tutuala-Mehara; } \\
\text { northern area }\end{array}$ & $\begin{array}{l}\text { Lorilata } \\
\text { Namilata }\end{array}$ & South of Ili Kere Kere. & $\begin{array}{l}\text { Walled site with cliffs } \\
\text { on eastern side. Has } \\
\text { graves, sepu ritual } \\
\text { ground and sacrificial } \\
\text { post (sikua). }\end{array}$ & $\begin{array}{l}\text { Tutuala Ratu defensive site. Former } \\
\text { fortified residence of Tutuala Ratu. } \\
\text { See Pannell (2006) for discussion. }\end{array}$ \\
\hline 15 & $\begin{array}{l}\text { Tutuala-Mehara; } \\
\text { northern area }\end{array}$ & $\begin{array}{l}\text { Hi Maka } \\
\text { Loli }\end{array}$ & $\begin{array}{l}\text { Lat. }-08.39967^{\circ} \\
\text { Long. } 127.26070^{\circ} \\
\text { Alt. } 410 \mathrm{~m} \\
\text { Prominent peaked hill } \\
\text { and sheer cliff south of } \\
\text { Tutuala kota. }\end{array}$ & $\begin{array}{l}\text { Fortified site with } \\
\text { internal graves and } \\
\text { porcelain scatters. } \\
\text { Front gateway } \\
\text { damaged. Fine } \\
\text { example of sepu. }\end{array}$ & $\begin{array}{l}\text { Owned by Vacumura Ratu. Origin } \\
\text { settlement before moving west. } \\
\text { All Vacumura Ratu once lived here. }\end{array}$ \\
\hline 16 & $\begin{array}{l}\text { Tutuala-Mehara; } \\
\text { northern area }\end{array}$ & $\begin{array}{l}\text { Cailoro } \\
\text { Lata }\end{array}$ & $\begin{array}{l}\text { Lat. }-08.39556^{\circ} \\
\text { Long. } 127.25703^{\circ} \\
\text { Alt. } 350 \mathrm{~m} \\
\text { Site is proximate to } \\
\text { local school in Tutuala } \\
\text { near the main church. }\end{array}$ & $\begin{array}{l}\text { Now largely } \\
\text { dismantled, rock of } \\
\text { the fort was used } \\
\text { to build the Tutuala } \\
\text { Pousada guest house } \\
\text { and police station } \\
\text { following Portuguese } \\
\text { pacification campaign } \\
\text { in } 1902 \text {. }\end{array}$ & $\begin{array}{l}\text { Formerly a major fortified site. War } \\
\text { skulls and tei altar in small cave } \\
\text { below western cliff of site. Cailoro } \\
\text { traded with Makassar at João on } \\
\text { the northern coast. }\end{array}$ \\
\hline 17 & $\begin{array}{l}\text { Tutuala-Mehara; } \\
\text { northern area }\end{array}$ & Наго & $\begin{array}{l}\text { Lat. }-08.23 .484^{\circ} \\
\text { Long. } 127.15 .493^{\circ} \\
\text { Alt. } 376 \mathrm{~m} \\
\text { Close to current Tutuala } \\
\text { Pousada. }\end{array}$ & $\begin{array}{l}\text { Fortified site with } \\
\text { damaged walls. } \\
\text { See Lape (2006) for } \\
\text { sequence and Pannell } \\
\text { (2006) for history } \\
\text { of use. }\end{array}$ & $\begin{array}{l}\text { Site of former residence of Tutuala } \\
\text { Ratu who were moved here in the } \\
\text { 1920s when the Portuguese built } \\
\text { the pousada. }\end{array}$ \\
\hline 18 & $\begin{array}{l}\text { Tutuala-Mehara; } \\
\text { northern areas }\end{array}$ & $\begin{array}{l}\text { Mapulu Ro } \\
\text { Malae }\end{array}$ & $\begin{array}{l}\text { Lat. }-08.41049^{\circ} \\
\text { Long. } 127.24127^{\circ} \\
\text { Alt. } 405 \mathrm{~m} \\
\text { (GPS for entrance } \\
\text { to inner walled } \\
\text { compound). } \\
\text { On forested hill at the } \\
\text { top of the Vero River } \\
\text { catchment. }\end{array}$ & $\begin{array}{l}\text { Fort has a series of } \\
\text { low outer ring walls } \\
\text { with standing stones } \\
\text { as defensive posts. } \\
\text { Massive inner walls } \\
\text { and extensive internal } \\
\text { spaces. }\end{array}$ & $\begin{array}{l}\text { Formerly three groups lived there: } \\
\text { Mapulo Ratu (Renu), Kiki Moru } \\
\text { (Koavaca Ratu) and a subsidiary } \\
\text { group, Ro Malae. Internal layout } \\
\text { reflects these divisions. }\end{array}$ \\
\hline 19 & Tutuala & Jasa Lata & $\begin{array}{l}\text { Lat. }-08.383117^{\circ} \\
\text { Long. } 127.238650^{\circ} \\
\text { Alt. } 274 \mathrm{~m}\end{array}$ & $\begin{array}{l}\text { Fortified settlement } \\
\text { with very large walls. } \\
\text { Visited with Pedro } \\
\text { Morais. }\end{array}$ & - \\
\hline 20 & $\begin{array}{l}\text { Tutuala-Mehara; } \\
\text { northern areas }\end{array}$ & Lo Chami & $\begin{array}{l}\text { Lat. }-08.388783^{\circ} \\
\text { Long. } 127.234683^{\circ} \\
\text { Alt. } 300 \mathrm{~m} \\
\text { On elevated ridge and } \\
\text { hilltop, south of Aldeia } \\
\text { lyoro. }\end{array}$ & $\begin{array}{l}\text { Fort not visited-not } \\
\text { permitted-they only } \\
\text { attend on All Souls' } \\
\text { day/night (i vaci } \\
\text { huma'ara). Walled site } \\
\text { with cliff on one side } \\
\text { (see Lape 2006). }\end{array}$ & $\begin{array}{l}\text { Ancestral site for Koavaca Ratu and } \\
\text { Paiuru Ratu and former residential } \\
\text { site during times of warfare. }\end{array}$ \\
\hline
\end{tabular}




\begin{tabular}{|c|c|c|c|c|c|}
\hline No. & Subdistrict & Site name & Location & Description & Notes \& significance \\
\hline 21 & $\begin{array}{l}\text { Tutuala-Jaco } \\
\text { Island }\end{array}$ & Lai Vai & $\begin{array}{l}\text { Lat. }-08.4398^{\circ} \\
\text { Long. } 127.3254^{\circ} \\
\text { Alt. } 50 \mathrm{~m} \\
\text { On southern area of Jaco } \\
\text { Island and c. } 700 \mathrm{~m} \\
\text { inland from coast. }\end{array}$ & $\begin{array}{l}\text { Fortified site. Adat } \\
\text { platform and tei } \\
\text { stone. Site visited } \\
\text { with Orlando Sanchez. }\end{array}$ & $\begin{array}{l}\text { 1st walled settlement on Jaco } \\
\text { Island. Zenlai Ratu accredited } \\
\text { with ownership of former fort } \\
\text { before moving to the mainland } \\
\text { (mua lafae, Timor). }\end{array}$ \\
\hline 22 & $\begin{array}{l}\text { Tutuala-Jaco } \\
\text { Island }\end{array}$ & Pitilete & 0n Jaco Island. & $\begin{array}{l}\text { Fortified site. 2nd } \\
\text { walled settlement } \\
\text { on Jaco Island after } \\
\text { Lai Vai. }\end{array}$ & $\begin{array}{l}\text { Zenlai Ratu accredited with } \\
\text { ownership of former fort } \\
\text { before moving to the mainland } \\
\text { (mua lafae, Timor). }\end{array}$ \\
\hline 23 & $\begin{array}{l}\text { Tutuala-Jaco } \\
\text { Island }\end{array}$ & Honolati & 0n Jaco Island. & $\begin{array}{l}\text { 4th walled settlement } \\
\text { on Jaco Island after } \\
\text { Lai Vai. }\end{array}$ & $\begin{array}{l}\text { Zenlai Ratu accredited with } \\
\text { ownership. }\end{array}$ \\
\hline 24 & $\begin{array}{l}\text { Tutuala-Valu- } \\
\text { Jaco }\end{array}$ & Lopo Malai & $\begin{array}{l}\text { Lat. }-08.407850^{\circ} \\
\text { Long. } 127.291567^{\circ} \\
\text { Alt. } 175 \mathrm{~m} \\
\text { Near Lene Hara Cave, } \\
\text { above Valu Beach. }\end{array}$ & $\begin{array}{l}\text { Crumbling remnant } \\
\text { fort. Small walled } \\
\text { compound. Much of } \\
\text { the stone has been } \\
\text { repurposed. }\end{array}$ & $\begin{array}{l}\text { Ma'a leki Ratu claims ownership } \\
\text { of area-(mua hocavaru) Ma'a Leki } \\
\text { and Zenlai Ratu controlled trading } \\
\text { ports at Mua Cao pasaré and Lopo } \\
\text { malar(U), (Valu Beach) respectively } \\
\text { and received hiaré (landing fees). } \\
\text { See Lape (2006). }\end{array}$ \\
\hline 25 & $\begin{array}{l}\text { Tutuala-Valu- } \\
\text { Jaco }\end{array}$ & Muacao & $\begin{array}{l}\text { Lat. }-08.40372^{\circ} \\
\text { Long. } 127.27949^{\circ} \\
\text { Alt. } 320 \mathrm{~m} \\
\text { Near Lene Нага. }\end{array}$ & $\begin{array}{l}\text { Crumbling circular } \\
\text { stone settlement. } \\
\text { Walls not intact } \\
\text { as stone has been } \\
\text { repurposed to make } \\
\text { garden walls. }\end{array}$ & $\begin{array}{l}\text { Zenlai Ratu moved to this place } \\
\text { when they left Lene Hara. }\end{array}$ \\
\hline 26 & $\begin{array}{l}\text { Tutuala-Vero } \\
\text { River valley, } \\
\text { southeast of } \\
\text { Paichao Range }\end{array}$ & Pati-patinu & $\begin{array}{l}\text { Lat. }-08.4707^{\circ} \\
\text { Long. } 127.2351^{\circ} \\
\text { Alt. } 100 \mathrm{~m} \\
\text { On elevated bluff } \\
\text { overlooking Jaco Island. }\end{array}$ & Not visited. & $\begin{array}{l}\text { Keveresi Ratu-now nearly died } \\
\text { out. Mythic place where the land } \\
\text { was divided among } 14 \text { groups } \\
\text { (Pannell pers. comm.) who had } \\
\text { settled from outside. }\end{array}$ \\
\hline 27 & $\begin{array}{l}\text { Tutuala-Vero } \\
\text { River valley, } \\
\text { southeast of } \\
\text { Paichao Range }\end{array}$ & $\begin{array}{l}\text { Haka Paku } \\
\text { Leki }\end{array}$ & $\begin{array}{l}\text { Lat. }-08.47427^{\circ} \\
\text { Long. } 127.21465^{\circ} \\
\text { Alt. } 248 \mathrm{~m} \\
\text { Limestone rocky outcrop } \\
\text { to the southeast of the } \\
\text { Paichao Range. }\end{array}$ & $\begin{array}{l}\text { Strategic semi-fortified } \\
\text { site; has extensive } \\
\text { elevated defensive } \\
\text { walls lower down } \\
\text { and rises to a narrow } \\
\text { shelf overlooking } \\
\text { the southeast coast. } \\
\text { Scatters of pottery on } \\
\text { highest points. } \\
\end{array}$ & $\begin{array}{l}\text { The mythical strategic fortress of } \\
\text { Renu Ratu, which dominated this } \\
\text { area of Ponta Leste. }\end{array}$ \\
\hline 28 & $\begin{array}{l}\text { Tutuala-Vero } \\
\text { River valley, } \\
\text { southeast of } \\
\text { Paichao Range }\end{array}$ & Ili Haraku & $\begin{array}{l}\text { Lat. }-08.48510^{\circ} \\
\text { Long. } 127.20291^{\circ} \\
\text { (GPS for inner wall near } \\
\text { the gateway.) } \\
\text { Alt. } 257 \mathrm{~m} \\
\text { On the upper slopes } \\
\text { to the immediate } \\
\text { southeast of Paichao } \\
\text { Range. }\end{array}$ & $\begin{array}{l}\text { Large fortified site } \\
\text { with extensive dry } \\
\text { walls. Large cliff face } \\
\text { to the east. Inner } \\
\text { and outer walls } \\
\text { with gateways. Site } \\
\text { inhabited in living } \\
\text { memory-probably } \\
\text { until World War II. }\end{array}$ & $\begin{array}{l}\text { Historically linked to Renu Ratu and } \\
\text { their warrior allies Aca Cao Ratu. } \\
\text { Recalls wars against Marapaki } \\
\text { and Zenlai Ratu who attacked the } \\
\text { fort. Renu leader killed, but Aca } \\
\text { Cao resisted and forces eventually } \\
\text { withdrew. Boundary on the coast } \\
\text { between Sere Moko and Sere Lafai. }\end{array}$ \\
\hline 29 & $\begin{array}{l}\text { Tutuala-Vero } \\
\text { River valley, } \\
\text { southeast of } \\
\text { Paichao Range }\end{array}$ & Serelau & $\begin{array}{l}\text { Located in hill between } \\
\text { coast and Paichao } \\
\text { Range, nearby and to } \\
\text { the north of Ili Haraku. }\end{array}$ & $\begin{array}{l}\text { Fortified site with } \\
\text { stone walls but not } \\
\text { sighted. Not visited. }\end{array}$ & $\begin{array}{l}\text { Current owners live in Aldeia } \\
\text { Vero (Tutuala). Serelau Ratu (see } \\
\text { Fitzpatrick et al. 2012). }\end{array}$ \\
\hline 30 & $\begin{array}{l}\text { Tutuala-Vero } \\
\text { River valley, } \\
\text { southeast of } \\
\text { Paichao Range }\end{array}$ & Maiana & $\begin{array}{l}\text { Lat. }-08.49096^{\circ} \\
\text { Long. } 127.19747^{\circ} \\
\text { Alt. } 300 \mathrm{~m} \\
\text { Located east of } \mathrm{Ili} \\
\text { Haraku as a free- } \\
\text { standing hill. }\end{array}$ & $\begin{array}{l}\text { Smaller fortified site } \\
\text { with circular walls } \\
\text { surrounding a large } \\
\text { stone (non-Christian) } \\
\text { grave. Massive walls } \\
\text { surrounded by prickly } \\
\text { pear cactus. }\end{array}$ & $\begin{array}{l}\text { Formerly Aca Cao Ratu granted to } \\
\text { Pai'ir Ratu. Large grave with fine } \\
\text { Makassar stone-dressed finish } \\
\text { (Makassar mataru) (see McWilliam } \\
\text { et al. 2012). Grave of a Paiuru Ratu } \\
\text { close to boundary between Renu } \\
\text { Ratu and Latuloho Ratu (Muapitine) } \\
\text { at the creek, Verkass Ver. }\end{array}$ \\
\hline
\end{tabular}




\begin{tabular}{|c|c|c|c|c|c|}
\hline No. & Subdistrict & Site name & Location & Description & Notes \& significance \\
\hline 31 & $\begin{array}{l}\text { Tutuala-Vero } \\
\text { River valley, } \\
\text { southeast of } \\
\text { Paichao Range }\end{array}$ & Pai Lopo & $\begin{array}{l}\text { Located to the south } \\
\text { of Maiana towards Ili } \\
\text { Mimiraka and boundary } \\
\text { with Pai Chao Ratu land. }\end{array}$ & Not sighted. & Aca Cao Ratu place. \\
\hline 32 & $\begin{array}{l}\text { Muapitine- } \\
\text { Malahara and } \\
\text { southern forests }\end{array}$ & Pari Loho & $\begin{array}{l}\text { Lat. }-08.485102^{\circ} \\
\text { Long. } 127.174104^{\circ} \\
\text { Alt. } 500 \mathrm{~m} \\
\text { Southwest of Malahara } \\
\text { settlement } 5-6 \mathrm{~km} \text { in } \\
\text { dense secondary forest. }\end{array}$ & $\begin{array}{l}\text { Extensive stone } \\
\text { walled fortified site } \\
\text { overgrown with large } \\
\text { Ficus trees. }\end{array}$ & $\begin{array}{l}\text { Part of the lands of Pai Chao Ratu. } \\
\text { Former inhabitants (Kua Mai Ratu) } \\
\text { left the area many years ago. Used } \\
\text { as jungle camp by both Falintil and } \\
\text { Indonesian TNI' soldiers during } \\
\text { occupation. }\end{array}$ \\
\hline 33 & $\begin{array}{l}\text { Muapitine- } \\
\text { Malahara and } \\
\text { southern forests }\end{array}$ & Veter(u) & $\begin{array}{l}\text { Located on 'little Pai } \\
\text { Chao'. Eastern side of } \\
\text { Paichao Range. }\end{array}$ & $\begin{array}{l}\text { Massive limestone } \\
\text { outcrop with steep } \\
\text { cliffs around much } \\
\text { of the structure and } \\
\text { stone walled sections. } \\
\text { Large double grave } \\
\text { site for sacrificial } \\
\text { veneration. }\end{array}$ & $\begin{array}{l}\text { Mythological landing site of Pai } \\
\text { Chao Ratu, seagoing perahu now } \\
\text { fossilised and embedded in the } \\
\text { land. To the south another walled } \\
\text { structure-known as Lamira- } \\
\text { former settlement of subsidiary } \\
\text { (Paca-Kanaluri) group. }\end{array}$ \\
\hline 34 & $\begin{array}{l}\text { Muapitine - } \\
\text { Malahara and } \\
\text { southern forests }\end{array}$ & Nofitu & $\begin{array}{l}\text { Lat. }-08.456269^{\circ} \\
\text { Long. } 127.195125^{\circ} \\
\text { Approx. GPS } \\
\text { Alt. } 680 \mathrm{~m} \\
\text { On prominent hill } \\
\text { between Lake Ira } \\
\text { La Laru and Pualoki } \\
\text { (Paichao mountains). }\end{array}$ & $\begin{array}{l}\text { Fortified sites. Not } \\
\text { visited. }\end{array}$ & $\begin{array}{l}\text { Mythic settlement associated with } \\
\text { Cailoro Ratu and referring to the } \\
\text { morning star (venus, noi ipi). } \\
\text { Another related site not located: } \\
\text { No Kafa. }\end{array}$ \\
\hline 35 & $\begin{array}{l}\text { Muapitine - } \\
\text { Malahara and } \\
\text { southern forests }\end{array}$ & $\begin{array}{l}\text { Mua Pitine } \\
\text { Irinu }\end{array}$ & $\begin{array}{l}\text { On upper reaches of } \\
\text { Aramoko creek. Located } \\
\text { in hills due south of } \\
\text { current Mua Pitine } \\
\text { settlement. }\end{array}$ & $\begin{array}{l}\text { Reported to be large } \\
\text { fortified site. Not } \\
\text { visited. }\end{array}$ & $\begin{array}{l}\text { Latu Loho Ratu is the land owner } \\
\text { (mua ho cavaru). When threatened } \\
\text { by } 1902 \text { Portuguese campaign, } \\
\text { surrendered without losses. } \\
\text { Remained inhabited for many years. }\end{array}$ \\
\hline 36 & $\begin{array}{l}\text { Muapitine- } \\
\text { Malahara and } \\
\text { southern forests }\end{array}$ & Pehe & $\begin{array}{l}\text { In elevated position } \\
\text { south coast, western } \\
\text { side of Aramoko creek. }\end{array}$ & $\begin{array}{l}\text { Not visited. The } \\
\text { fort is an ancestral } \\
\text { settlement of current } \\
\text { members of Lupuloho } \\
\text { hamlet in Suco } \\
\text { Muapitine. }\end{array}$ & $\begin{array}{l}1908 \text { residents relocated to a } \\
\text { road connecting to Lorehe and } \\
\text { then moved en masse to Los } \\
\text { Palos in } 1976 \text { by Indonesian } \\
\text { army. Key owners are Pui Rili, } \\
\text { Aca Cao, Paraluki and Naja Ratu. } \\
\text { People still attend rituals and } \\
\text { annual meci (sea worm) harvest } \\
\text { there-footpath to Muapitine } \\
\text { (see McWilliam 2007b). }\end{array}$ \\
\hline 37 & $\begin{array}{l}\text { Muapitine-Los } \\
\text { Palos and } \\
\text { southern forests }\end{array}$ & Belta Tres & $\begin{array}{l}\text { In hills } 4 \text { km south of } \\
\text { Los Palos. }\end{array}$ & $\begin{array}{l}\text { Fortified site. Not } \\
\text { visited, Fataluku } \\
\text { owners now resident } \\
\text { in urban Aldeia of Los } \\
\text { Palos, known as Ira } \\
\text { Ara. Relocated during } \\
\text { occupation. }\end{array}$ & $\begin{array}{l}\text { Members of Ira Ara were staunch } \\
\text { supporters of independence } \\
\text { and active in armed resistance. } \\
\text { Fortified site remains an important } \\
\text { place for refuge and sacrificial } \\
\text { veneration. }\end{array}$ \\
\hline 38 & $\begin{array}{l}\text { Muapitine-Los } \\
\text { Palos and } \\
\text { southern forests }\end{array}$ & Lereloho & $\begin{array}{l}\text { In the forested hills } \\
3-4 \text { km south of Los } \\
\text { Palos. }\end{array}$ & $\begin{array}{l}\text { Fortified site. Not } \\
\text { visited. Fataluku } \\
\text { owners now resident } \\
\text { in urban Aldeia of } \\
\text { Lereloho. Relocated } \\
\text { during occupation. }\end{array}$ & $\begin{array}{l}\text { Members of Lereloho active } \\
\text { in independence struggle and } \\
\text { clandestine resistance. Fortified } \\
\text { site and general area still regularly } \\
\text { frequented. }\end{array}$ \\
\hline
\end{tabular}

Note: ${ }^{1}$ TNI = Tentara Nasional Indonesia, the Indonesian National Military.

Sources: Authors' summary, see also references throughout table. 


\section{Fortified sites on the island of Kisar, Maluku Barat Daya, Indonesia}

Kisar was surveyed over two field seasons in 2014 and 2015 by a joint team from ANU, Universitas Gadjah Mada and Balar Arkeologi Maluku. A number of abandoned fortifications were located in open elevated positions, as well as in caves/shelters, during archaeological reconnaissance of the island (Figure 11.12; Table 11.3).

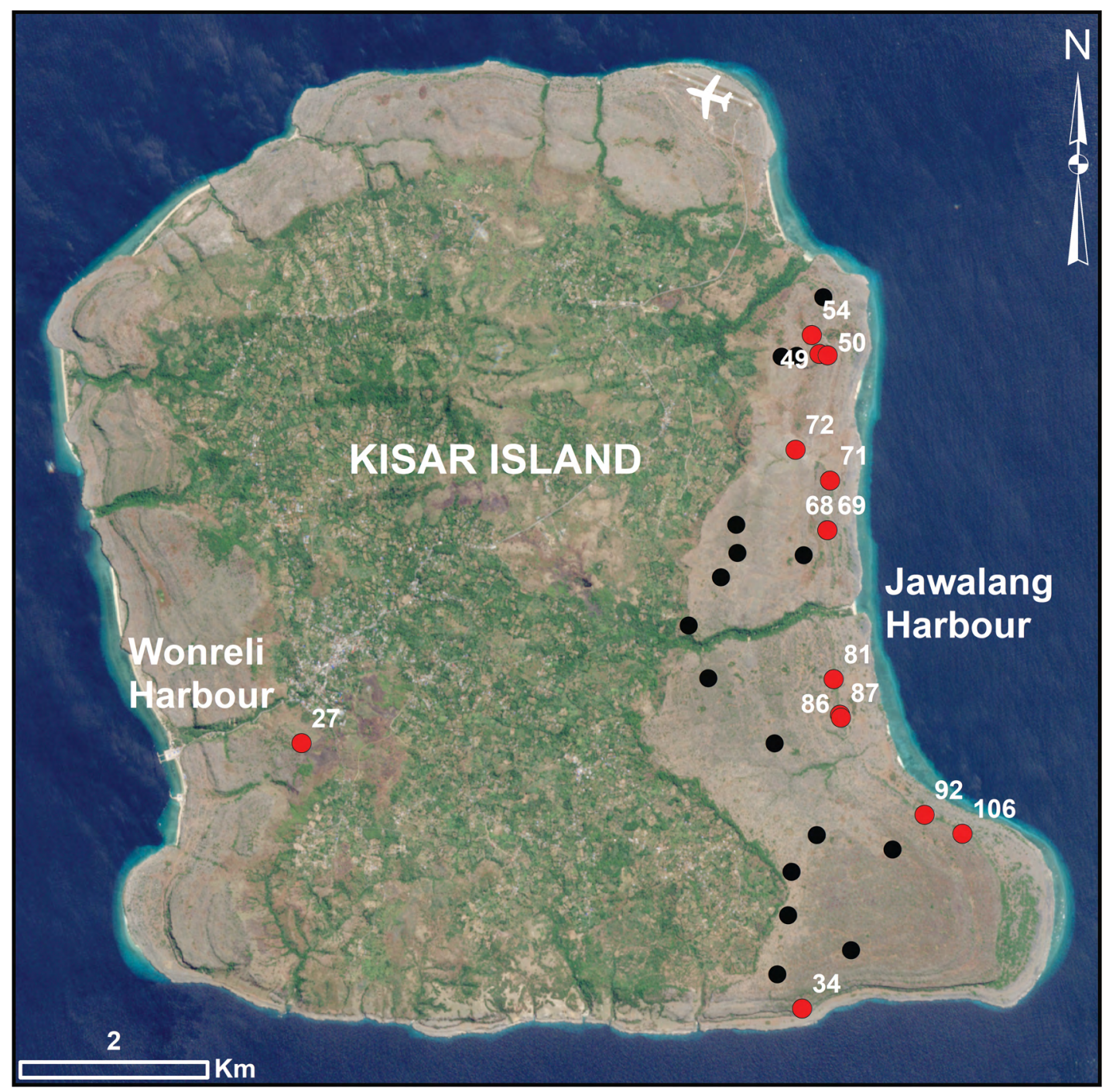

Figure 11.12. Map showing the location of identified fort sites on Kisar island.

Sites identified during pedestrian survey of the island are shown in red and numbered based on their KSR codes (see Table 11.3). Likely additional fort sites identified during remote-sensing surveys (but not yet ground truthed) are shown in black.

Source: Satellite image from the Sentinel-2A satellite (2019-07-10) obtained from the Sentinel Data Hub (European Space Agency 2019).

In 2014, five fortified sites were recorded in Kisar: KSR 27, 34, 49, 50 and 54. KSR 27 is located on the highest point of the hill overlooking the modern town of Wonreli to the north and Wonreli Harbour to the west. Although it would once have had impressive walls, only remnants of these remain today. The walls appear to follow the highest points of the contour of the hill. Local informants stated that the stone from the walls had been reused for other structures after 
the settlement was abandoned. Features which are probable graves and remnant stone house bases were observed within the walls; however, much of the surface of the hilltop was obscured by dense vegetation.

KSR 34 is a cave that contains a stone altar. Oral history indicates that it was used as a defensive location by local people until World War II, although there is no evidence of stone walling to support this.

KSR 49 is a very large fortification (c. $0.02 \mathrm{~km}^{2} />15,000 \mathrm{~m}^{2}$ ) in an open hilltop location (Figure 11.13) with massive fortified stone walls (Figure 11.14). Based on local reports and our satellite survey of the island, KSR 49 is likely to be the largest fortification of its type preserved on the island. Known as the Pur Pura Negeri Lama, it is said to be the original location of the Pur Pura (or Pura Pura) village, now located about $2 \mathrm{~km}$ inland to the west. The Pur Pura Negeri Lama is situated atop a bastion of the third limestone terrace (Figure 11.13A). Approximately half to three-quarters of the walls are built along the edge of the terrace cliffs, which rise c. $10 \mathrm{~m}$ above the plain and thus are naturally protected by the steep drop-off in elevation (Figure 11.13B). The walls extending up from the cliff edge are similar in construction to those of the Negeri Lama visible to the south (KSR 72), but reach heights of c. $2 \mathrm{~m}$. As well as KSR 72, another fortification slightly to the southwest was clearly visible, however, due to time constraints we were unable to visit it. Later satellite surveys strongly suggest a similarity in design between this Negeri Lama and KSR 49 and 72 (see Figure 11.12 remotely sensed sites).
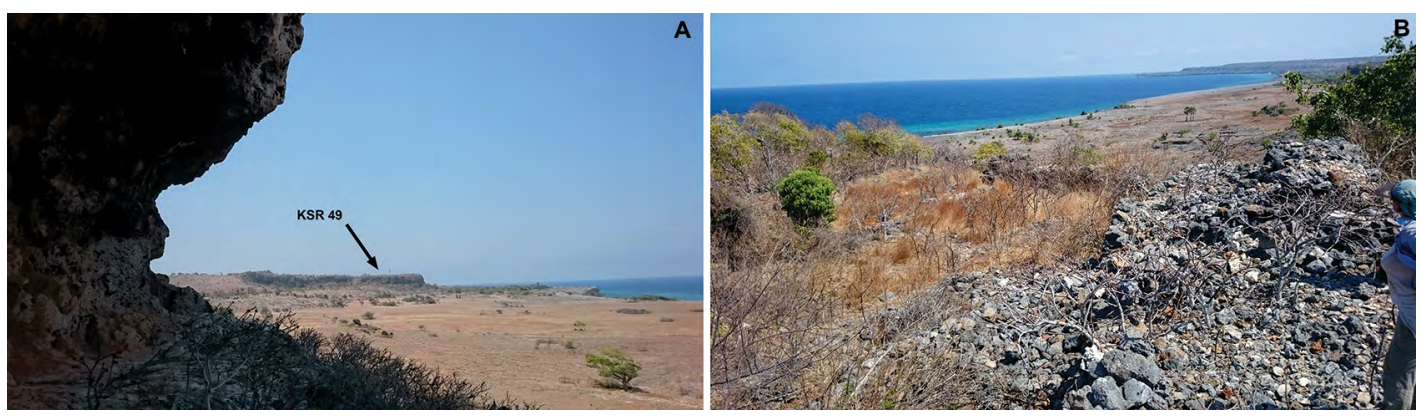

Figure 11.13. The view from Loi Puru Ula looking north (left) towards the Pur Pura Negeri Lama (KSR 49-indicated), and the view from the Pur Pura Negeri Lama south towards Loi Puru Ula (KSR 68 and 69).

Source: Photos courtesy of Shimona Kealy.

Along the portion of the Pur Pura plateau not edged by steep cliffs, dramatically larger walls have been built (Figure 11.14). Not only do these stone walls reach heights of up to c. $4 \mathrm{~m}$, but they are between 1 and $5 \mathrm{~m}$ thick and contain chamber-like areas within the walls (see Figure 11.14C). Narrow passageways allow entrance to the central part of the settlement (Figure 11.14A and D), which contains numerous stone features. For example, KSR 50 is a megalithic complex of shaped and dressed stones positioned in the centre of the KSR 49 fortification, but was recorded as an individual site, as it was enclosed by a separate walled enclosure. The complex includes collapsed menhir-like standing stones (Figure 11.15), and large circular stones of a fine-grained sedimentary rock, which are shaped and dressed and remarkably similar in shape and size to those found in fortified settlements in neighbouring Timor-Leste (McWilliam et al. 2012). 

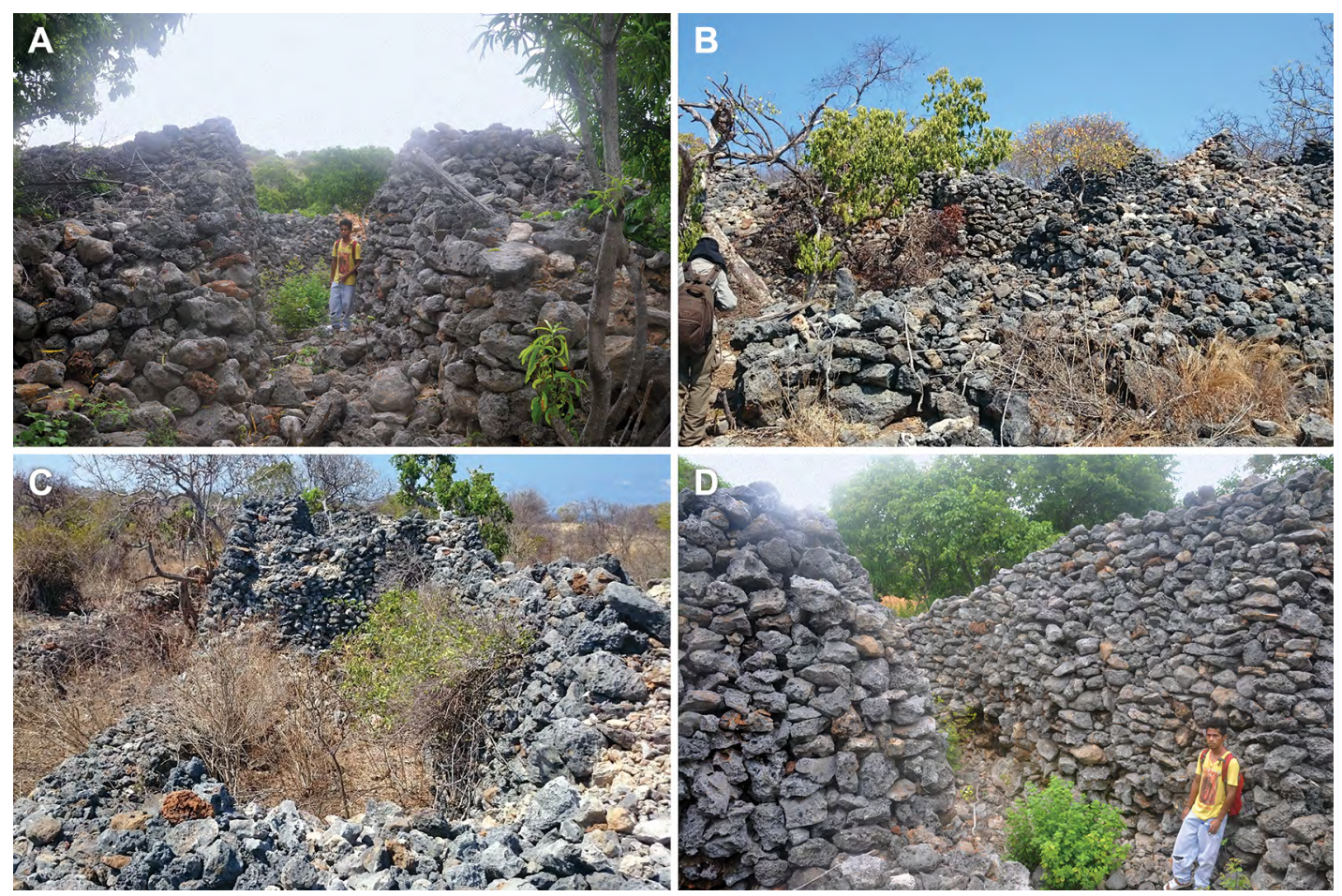

Figure 11.14. The Pur Pura Negeri Lama (KSR 49) showing the significant heights of the walls (A, D); extensive stonework (B) and chambers within the main wall dividing the headland (C).

Source: Photos A and D courtesy of Marlon Ririmasse; photos B and C courtesy of Shimona Kealy.

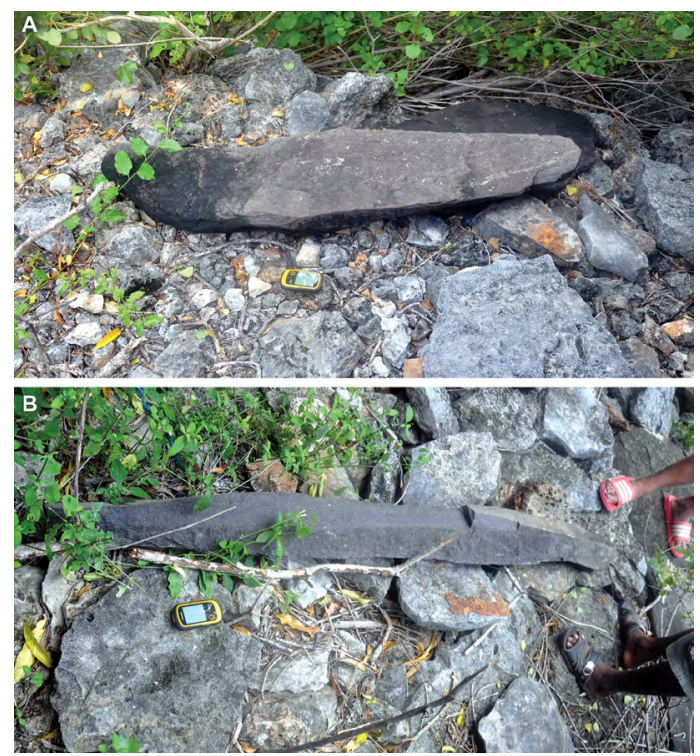

Figure 11.15. Fallen megalithic standing stones from the old Pur Pura village complex (KSR 50), located within the Pur Pura Negri Lama fortifications (KSR 49).

Source: Photos courtesy of Marlon Ririmasse.
KSR 54 is a very large cave extending into the same limestone terrace upon which KSR 49 is constructed (Figure 11.16). It contains the remnants of carved wooden posts (Figure 11.16B) which were said by our local guides to be part of an old fortified village, however it was unclear if these structures were built within the cave itself or came from the open fortified village KSR 49. KSR 54 did not have evidence of thick walling suggestive of a fortification. 

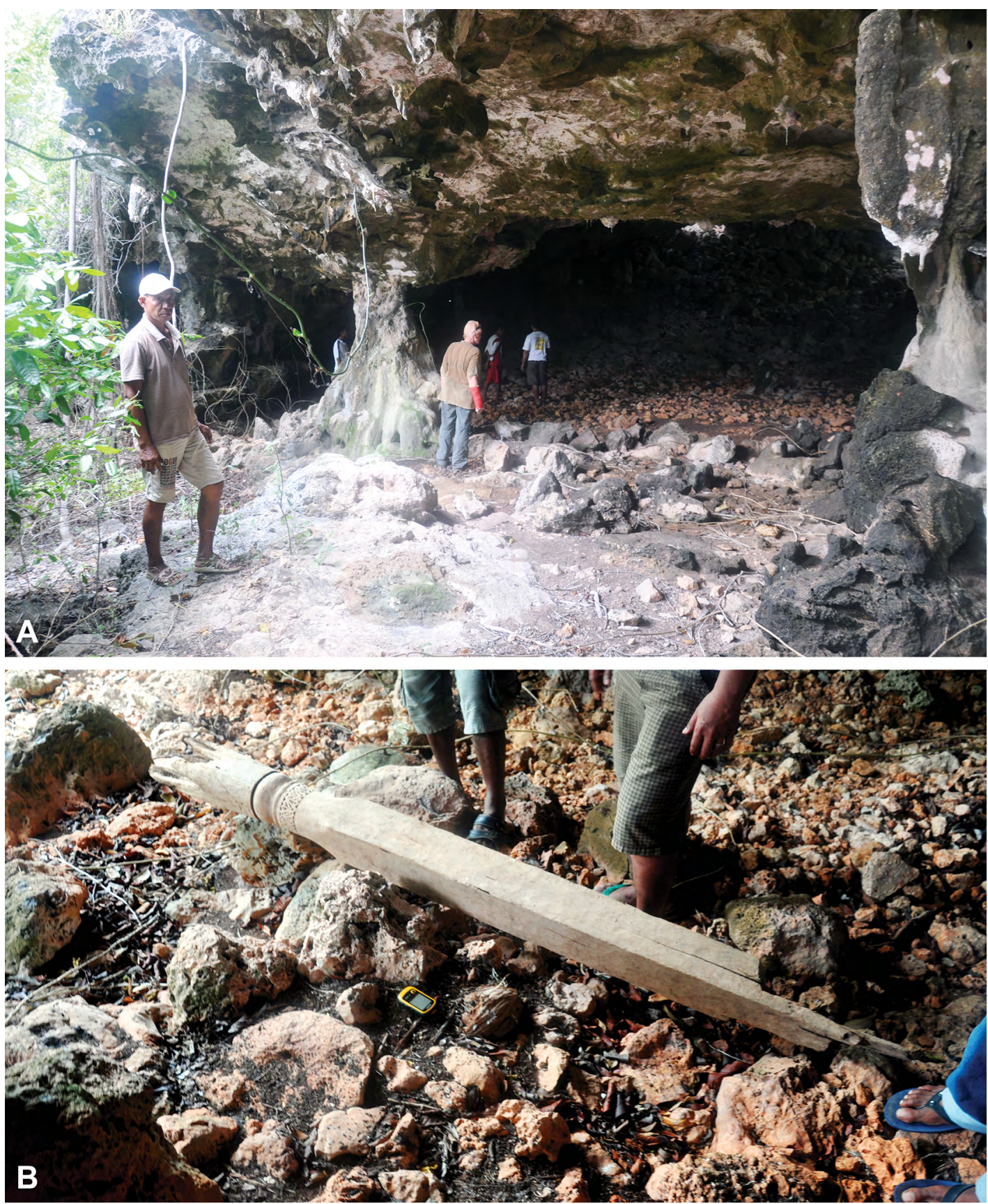

Figure 11.16. Cave (KSR 54) (A) below the Pur Pura Negri Lama containing carved timber post (B). Source: Photos courtesy of Marlon Ririmasse.

Located just over $600 \mathrm{~m}$ north of the Jawalang rock art locality (O'Connor et al. 2018) and $860 \mathrm{~m}$ north-northwest of Jawalang Harbour are several fortifications in the Loi Puru Ula locality (KSR 68, 69 and 71). This area consists of a series of largely interconnecting limestone shelters that are located approximately $4 \mathrm{~m}$ up the cliff face of the limestone terrace. The floors of these shelters, a number of walls and connecting gateways, and a remarkable staircase to access them all, are positioned at the entrance of KSR 68 (Figure 11.17). The walling and staircase have been constructed from significant quantities of stonework. The staircase, in particular, rises approximately $4 \mathrm{~m}$ to reach the shelter floor, is about $1 \mathrm{~m}$ wide, and extends an estimated $3 \mathrm{~m}$ along the face of the cliff. 

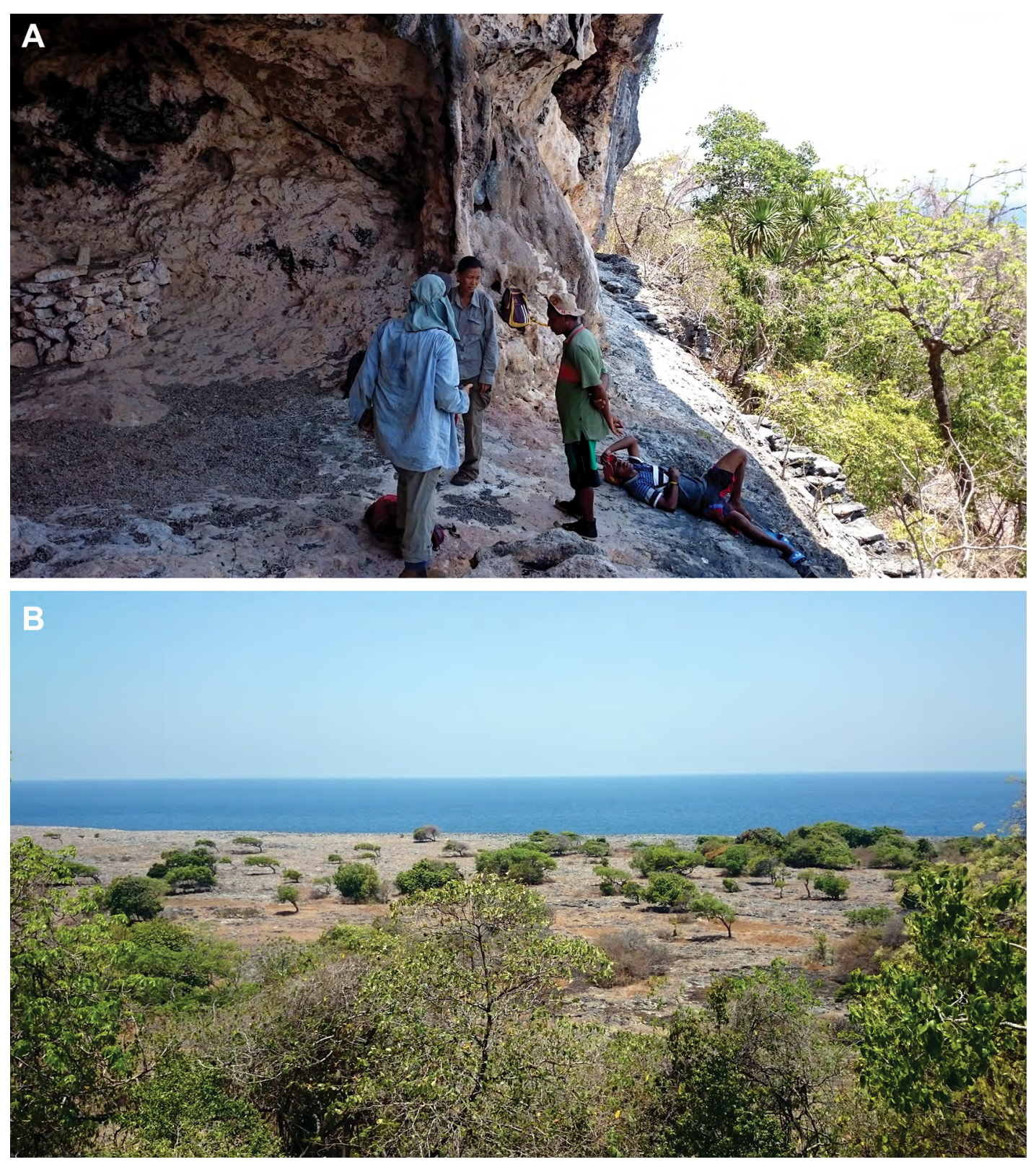

Figure 11.17. Loi Puru Ula 1 (KSR 68) fortified rock-shelter with altar (A: viewer's left) and rock wall from base of cliff to the lip of the shelter floor, forming a staircase for access to the site (A: viewer's right). The rock-shelter has a commanding view of the plain below (B).

Source: Photos courtesy of Shimona Kealy.

For the purpose of discussion, we have split the Loi Puru Ula site into its three main shelters, the southern (KSR 68), central (KSR 69) and northern shelters (KSR 71), which are each identifiable by their size and the presence of a stone altar. These three are, however, connected or semiconnected by narrow ledges and smaller shelters. The staircase rises to the floor of the southern shelter and then extends as a raised platform that broadens the shelter floors to the north. Within the southern shelter is a stone altar, built as a semicircular stone wall in the north corner, with a small pillar in the centre of an earth platform (Figure 11.17A). This altar still appears to be of significance today as our guides spent a moment in consultation when we first entered the site, before proceeding to show us around. 
Travel to the central shelter (KSR 69) is made significantly easier through the extension of the top of the staircase to the north (Figure 11.18A). This remarkable construction is made from a large accumulation of stone in a wall up the almost vertical cliff of the terrace to the lip of the shelter floor, thereby extending the internal living space. At the floor level, large naturally flat slabs of stone have been placed so as to enlarge the pathway and interconnected concavities of the shelters by about $1 \mathrm{~m}$ of flat paved floor. There is a stone wall approximately $0.5 \mathrm{~m}$ high separating the central and northern shelters with a small, stepped gateway built-in to provide access between the two (Figure 11.18B). The northern shelter is most notable for the large altar present, constructed of a single large stone slab approximately $1 \mathrm{~m}^{2}$ positioned atop smaller stone supports. The surface of the altar has a few small pits, likely formed as a result of grinding (e.g. nuts). The northern most extent of the Loi Puru Ula shelters ends in a squared stone wall about $0.5 \mathrm{~m}$ high.

Heading north from the Loi Puru Ula locality, there are a number of hills rising out of an open plain between Loi Puru Ula and the Pur Pura Negeri Lama (Figure 11.13). Approximately $750 \mathrm{~m}$ north-northwest of Loi Puru Ula (as the crow flies), situated on the top of one of the central hills, is the remains of an old fortification known as Nomaha Negeri Lama (KSR 72; Figure 11.19). Oral tradition states that this is the old village of Nomaha, now located about $1.5 \mathrm{~km}$ inland to the west. A stone wall around $1 \mathrm{~m}$ in height follows the topography, and completely encircles the hilltop (Figure 11.19B). Although small in size $\left(\right.$ c. $\left.650 \mathrm{~m}^{2}\right)$, this centrally located hill has a commanding view of the plain, while its steep sides, topped by the final obstacle of a stone wall, makes access difficult and would have presumably provided its inhabitants with a significant defensive advantage in the event of an attack (Figure 11.19A). From the Nomaha Negeri Lama, there is a view north (c. $1 \mathrm{~km}$ ) to Pur Pura Negeri Lama (KSR 49).

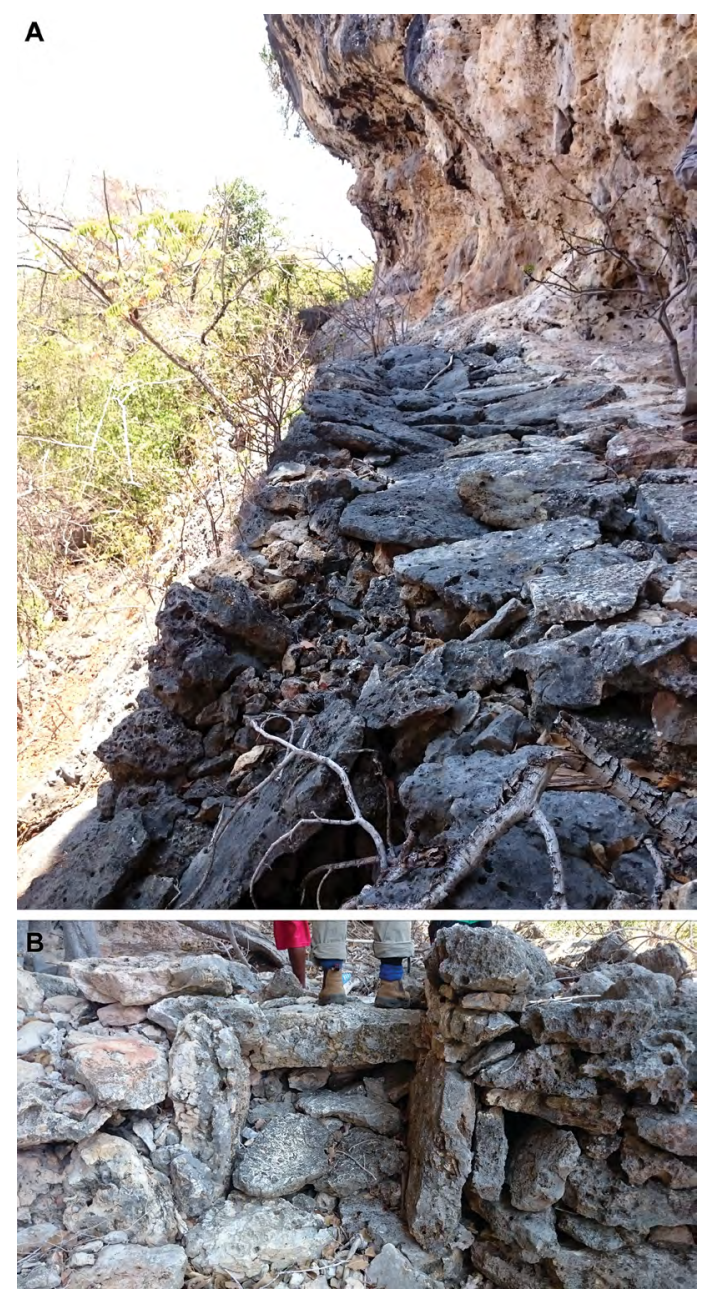

Figure 11.18. Loi Puru Ula 2 (KSR 69) showing the paved extended shelter floor (A) and stone wall with gate separating the different shelters along the terrace (B).

Source: Photos courtesy of Shimona Kealy. 


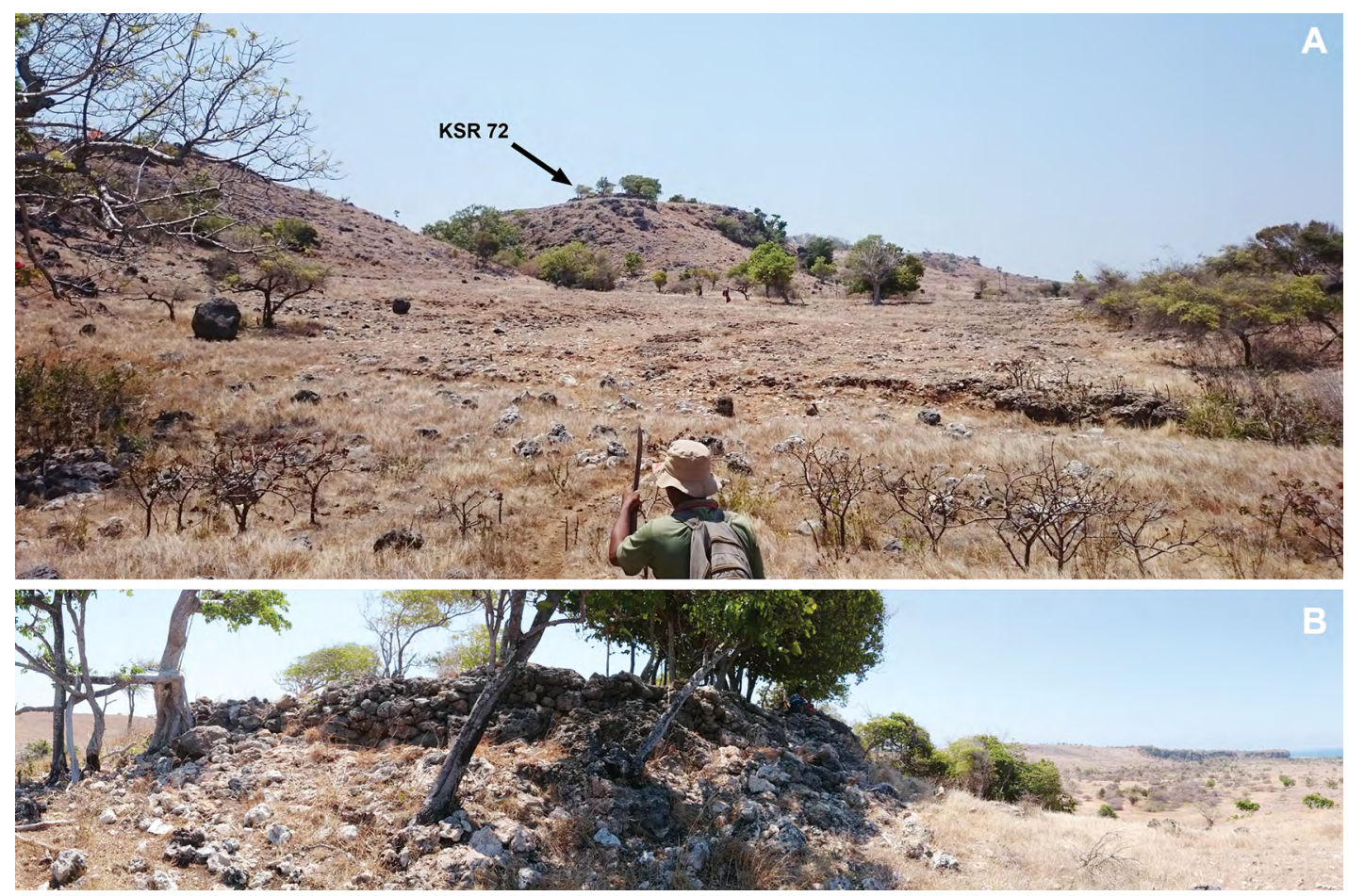

Figure 11.19. Nomaha Negeri Lama (KSR 72) view from plain looking north-northwest to the hilltop where the fortification is located $(A)$, and the view of the stone wall looking north (B).

Source: Photos courtesy of Shimona Kealy.

South of Jawalang Harbour following the terrace, a series of caves and fortifications were identified during surveys undertaken in 2015. The most northerly of these, Kota Lama 1 (KSR 81), consisted of a cave with fortifications enclosing the northern and southern ridgeline of a $-30 \times 10 \mathrm{~m}$ extended area of the terrace platform in front of the cave (Figure 11.20). Like many of these fortifications, the site overlooks a large area of the plain below (Figure 11.20B).

Approximately $400 \mathrm{~m}$ south of KSR 81, one of the cave sites, Worletiwuru (KSR 86), is partially fortified through the construction of a stone wall. On the south side of the cave a built stone wall forms the northern side of another fortified platform similar to KSR 81, which the locals identified by the name 'Sokon' (KSR 87). The southern walls of Sokon were particularly interesting for the zig-zag pattern by which the walls were constructed to maximise alignment to the terrace ridge (Figure 11.21).

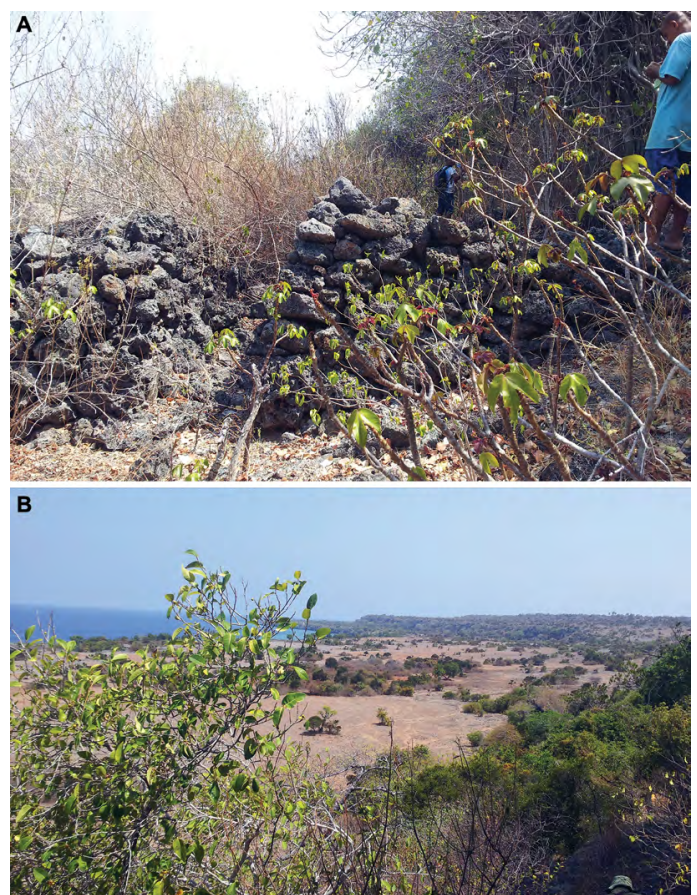

Figure 11.20. Jawalang Selatan Kota Lama 1 (KSR 81) showing stone wall with entrance (A) and the commanding view of the plain to the south-southeast (B).

Source: Photos courtesy of Stuart Hawkins. 

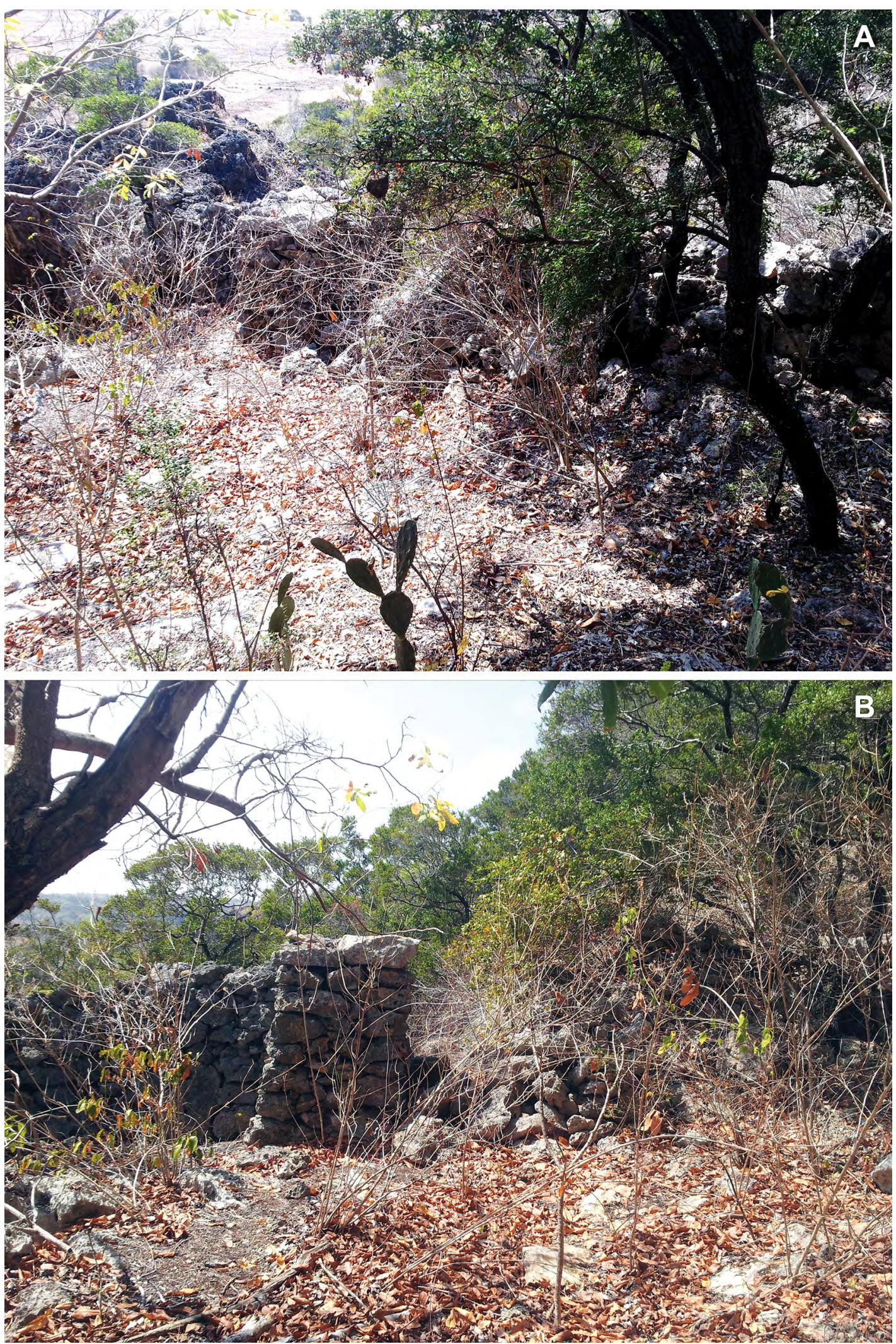

Figure 11.21. Sokon (KSR 87) fortification showing stone walls following the ridge (A) with ' $\mathrm{Z}$-shaped constructions (B, in detail).

Source: Photos courtesy of Stuart Hawkins. 


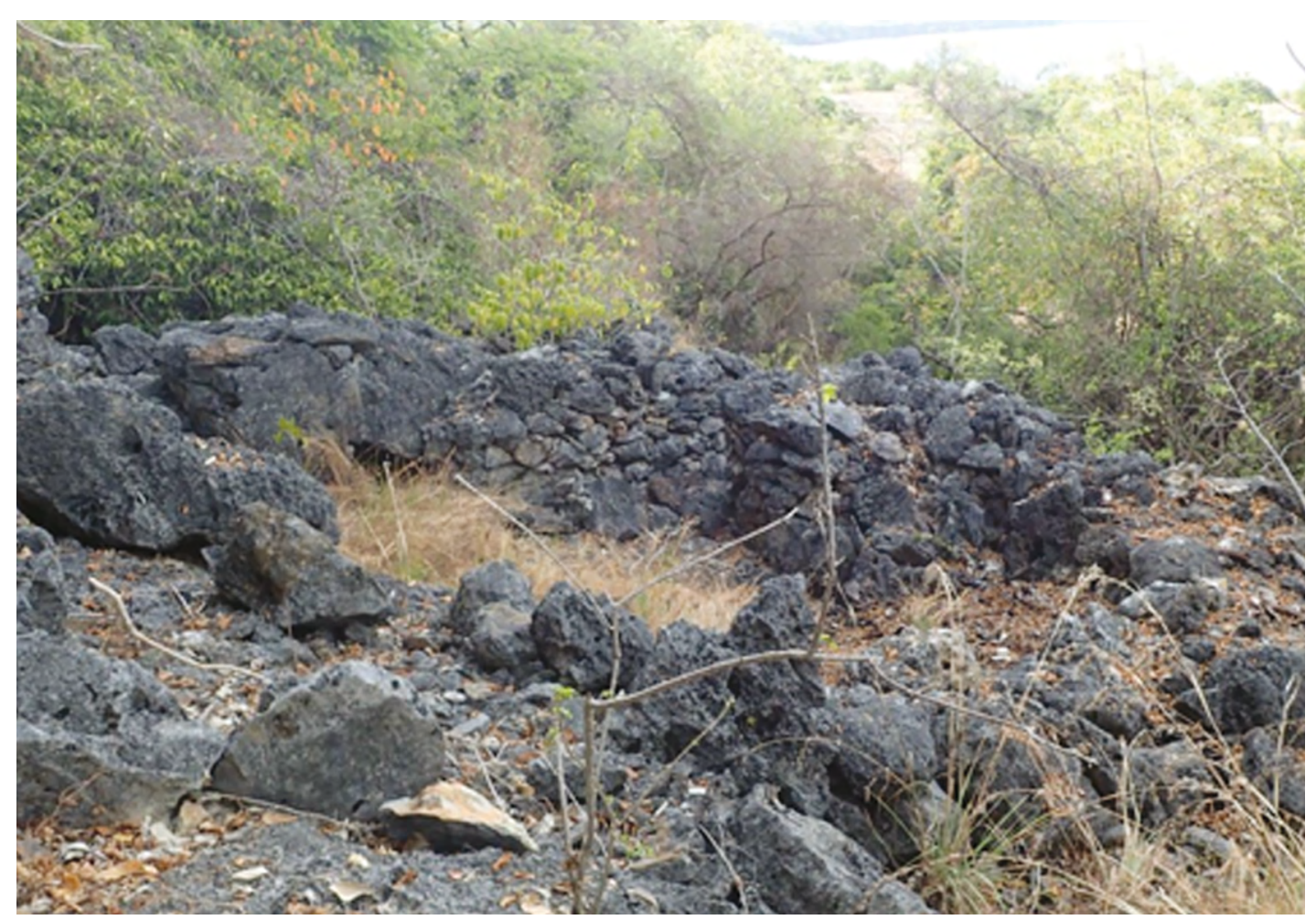

Figure 11.22. Jawalang Selatan Kota Lama 2 (KSR 106).

Source: Photo courtesy of Stuart Hawkins.

Further south from KSR 86 and 87, the terrace cliff curves slightly from an easterly facing aspect to a more northerly one. It is along here that Ilese Weraa (KSR 92) and Jawalang Selatan (south) Kota Lama 2 (KSR 106) were located, approximately $450 \mathrm{~m}$ apart. Ilese Weraa is an elevated cave with stone arrangements, while KSR 106 is a small, circular fortification on the terrace edge, built of uniformly dark stone (Figure 11.22) and located less than $1 \mathrm{~km}$ from the southeastern tip of the island.

Kisar is particularly promising for future fort survey and archaeological study as remote survey of the island using satellite imagery in Google Earth Pro V 7.3.2.5491 (2018a) identified at least 16 additional sites that match the unique features of the open Negeri Lamas identified during the brief ground surveys (see Figure 11.12 remotely sensed sites).

Table 11.3. Summary data on fortified sites in Kisar.

\begin{tabular}{|l|l|l|l|l|l|}
\hline${ }^{1}$ KSR No. & Region & Site name & Location & Description & Findings \\
\hline 27 & Wonreli Hill & $\begin{array}{l}\text { Negeri Lama } \\
\text { Katrow Wakrow }\end{array}$ & $\begin{array}{l}\text { Lat. }-08.07879^{\circ} \\
\text { Long. } 127.1603^{\circ} \\
\text { 2Alt. } 121 \mathrm{~m}\end{array}$ & $\begin{array}{l}\text { Old fortified } \\
\text { settlements }\end{array}$ & $\begin{array}{l}\text { Grave structure, fragments } \\
\text { of pottery. }\end{array}$ \\
\hline 34 & Oirata & Oirata Cave & $\begin{array}{l}\text { Lat. }-08.10466^{\circ} \\
\text { Long. } 127.2087^{\circ} \\
\text { Alt. } 44 \mathrm{~m}\end{array}$ & Cave with altar & $\begin{array}{l}\text { Oral history says this was occupied } \\
\text { until World War II. }\end{array}$ \\
\hline 49 & $\begin{array}{l}\text { Pur Pura } \\
\text { Village }\end{array}$ & $\begin{array}{l}\text { Pur Pura Negeri } \\
\text { Lama }\end{array}$ & $\begin{array}{l}\text { Lat. }-08.04156^{\circ} \\
\text { Long. } 127.2112^{\circ} \\
\text { Alt. } 54 \mathrm{~m}\end{array}$ & Fortified village & Gate with stone walls. \\
\hline 50 & $\begin{array}{l}\text { Pur Pura 0ld } \\
\text { Village }\end{array}$ & Megaliths & $\begin{array}{l}\text { Lat. }-08.04152^{\circ} \\
\text { Long. } 127.2115^{\circ} \\
\text { Alt. } 54 \mathrm{~m}\end{array}$ & $\begin{array}{l}\text { Erected stone and } \\
\text { dolmen complex }\end{array}$ & - \\
\hline
\end{tabular}




\begin{tabular}{|c|c|c|c|c|c|}
\hline${ }^{1}$ KSR No. & Region & Site name & Location & Description & Findings \\
\hline 54 & $\begin{array}{l}\text { Below Pur } \\
\text { Pura Negeri } \\
\text { Lama }\end{array}$ & $\begin{array}{l}\text { Pur Pura Negeri } \\
\text { Lama Cave } 2\end{array}$ & $\begin{array}{l}\text { Lat. }-08.03954^{\circ} \\
\text { Long. } 127.21^{\circ} \\
\text { Alt. } 49 \mathrm{~m}\end{array}$ & $\begin{array}{l}\text { Large cave below Pur } \\
\text { Pura Negeri Lama }\end{array}$ & $\begin{array}{l}\text { Wood statue said to be part of the } \\
\text { old fortified village. }\end{array}$ \\
\hline 68 & Loi Puru & Loi Puru Ula 1 & $\begin{array}{l}\text { Lat. }-08.05843^{\circ} \\
\text { Long. } 127.2114^{\circ} \\
\text { Alt. } 39 \mathrm{~m}\end{array}$ & Fortified shelter & $\begin{array}{l}\text { Stone walls, staircase and altar } \\
\text { with stone upright. Said to be used } \\
\text { for defence against raiding clans. }\end{array}$ \\
\hline 69 & Loi Puru & Loi Puru Ula 2 & $\begin{array}{l}\text { Lat. }-08.05843^{\circ} \\
\text { Long. } 127.2114^{\circ} \\
\text { Alt. } 39 \mathrm{~m}\end{array}$ & Fortified shelter & $\begin{array}{l}\text { Stone floor, stone altar, stone gate } \\
\text { with step. }\end{array}$ \\
\hline 71 & Loi Puru & Loi Puru Ula 4 & $\begin{array}{l}\text { Lat. }-08.05362^{\circ} \\
\text { Long. } 127.2117^{\circ} \\
\text { Alt. } 40 \mathrm{~m} \\
\end{array}$ & Fortified shelter/cave & $\begin{array}{l}\text { Evidence of habitation, such as } \\
\text { marine shell and stone wall built } \\
\text { inside cave. }\end{array}$ \\
\hline 72 & Loi Puru & $\begin{array}{l}\text { Nomaha Negeri } \\
\text { Lama }\end{array}$ & $\begin{array}{l}\text { Lat. }-08.05062^{\circ} \\
\text { Long. } 127.2083^{\circ} \\
\text { Alt. } 54 \mathrm{~m} \\
\end{array}$ & Negeri Lama & $\begin{array}{l}\text { The old fortified settlement } \\
\text { of Nomaha. }\end{array}$ \\
\hline 81 & $\begin{array}{l}\text { Jawalang } \\
\text { Harbour South }\end{array}$ & Kota Lama 1 & $\begin{array}{l}\text { Lat. }-08.07283^{\circ} \\
\text { Long. } 127.2119^{\circ} \\
\text { Alt. } 43 \mathrm{~m}\end{array}$ & Negeri Lama & Fortified ridge. \\
\hline 86 & $\begin{array}{l}\text { Jawalang } \\
\text { Harbour South }\end{array}$ & Worleti-wuru & $\begin{array}{l}\text { Lat. }-08.07628^{\circ} \\
\text { Long. } 127.2125^{\circ} \\
\text { Alt. } 41 \mathrm{~m} \\
\end{array}$ & Fortified cave & Stone wall. \\
\hline 87 & $\begin{array}{l}\text { Jawalang } \\
\text { Harbour South }\end{array}$ & Sokon & $\begin{array}{l}\text { Lat. }-08.07656^{\circ} \\
\text { Long. } 127.2126^{\circ} \\
\text { Alt. } 40 \mathrm{~m}\end{array}$ & Negeri Lama & Fortified ridge. \\
\hline 92 & $\begin{array}{l}\text { Jawalang } \\
\text { Harbour South }\end{array}$ & Ilese Weraa & $\begin{array}{l}\text { Lat. }-08.08599^{\circ} \\
\text { Long. } 127.2207^{\circ} \\
\text { Alt. } 40 \mathrm{~m}\end{array}$ & Elevated cave & Stone arrangements. \\
\hline 106 & $\begin{array}{l}\text { Jawalang } \\
\text { Harbour South }\end{array}$ & Kota Lama 2 & $\begin{array}{l}\text { Lat. }-08.08782^{\circ} \\
\text { Long. } 127.2244^{\circ} \\
\text { Alt. } 41 \mathrm{~m}\end{array}$ & Small fort & $\begin{array}{l}\text { Small, circular fortification near } \\
\text { terrace edge. }\end{array}$ \\
\hline
\end{tabular}

Notes: ${ }^{1}$ The KSR numbers refer to the complete archaeological survey of the island, thus some numbers are missing from this sequence. ${ }^{2}$ Approximate altitude.

Source: Authors' summary.

\section{The Babar Island group}

Fortified settlements in the Babar Island group were located by a joint ANU and Balar Arkeologi Maluku team composed of Sue O'Connor, Shimona Kealy and Lucas Wattimena during fieldwork in October 2017 (Table 11.4). The survey was not specifically aimed at locating fortified settlements but was a general reconnaissance for archaeological sites (Kealy et al. 2018). Old fortified settlements in this group were referred to by local guides and landowners as Negeri Lama. One potential Negeri Lama locality was identified atop the north plateau on Wetang island, based on the presence of stone structures and because of its location (\#6). A number of larger, significantly better preserved, Negeri Lama sites were identified on Babar Besar Island, all on the east coast with a single exception near the village of Manuwuy in the north (Figure 11.23). The forts have thick stone walls, sometimes up to $1.5 \mathrm{~m}$ wide and reaching $2 \mathrm{~m}$ high with entrance and exit 'gateways'. The walls of the forts are of varying height; those close to modern settlements and garden areas have often had stone from the walls removed to make modern garden walls or for other construction. The site near Manuwuy (\#27) is the clearest example of this and thus the most poorly preserved of the Negeri Lamas visited in the course of the Babar group survey. 


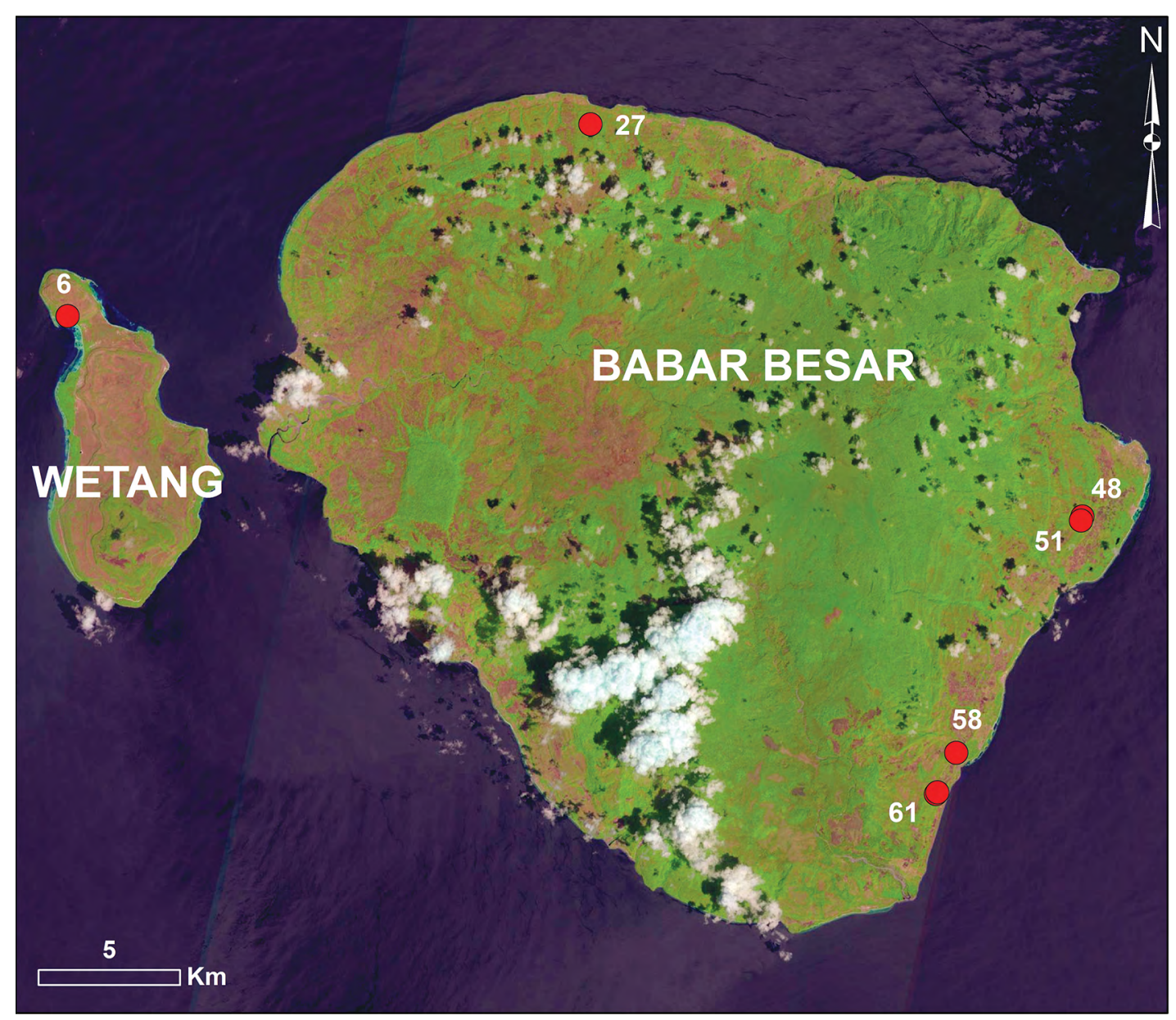

Figure 11.23. Map showing the location of identified fort sites on the islands of Wetang and Babar Besar. Sites are numbered based on Kealy et al. (2018) (see also Table 11.4).

Source: Satellite image from the Sentinel-2A satellite (2019-12-04) obtained from the Sentinel Data Hub (European Space Agency 2019).

The formation of the fort walls is roughly semicircular, with a steep escarpment or drop-off on the unwalled section of the construction providing natural defence. Oral history from local villagers records unanimously that these village settlements were occupied by individual clan groups. They were fortified with high walls for protection against raiding by other clan groups living in similar fortified settlements. In this part of the island, pacification and movement to the current coastal village is said to have occurred with the arrival of missionaries from the German Protestant Bremman Mission in 1917. A centennial celebration in honour of this occasion had taken place a few weeks before we began our survey. Oral history for multiple villages/clans in east Babar Besar begins with the occupation of a few ancestral villages high in the mountains. War and population expansion over time was said to have resulted in the movement of these villages, and sometimes the division of a village into two separate clans that then constructed individual 'new' fortifications on the adjacent lower terrace (closer to the coast). Repetition of this process over time resulted in a series of abandoned fortified villages stepping up into the hills. The modern distribution of present-day coastal villages is a result of pacification, with some clan groups recombining in the larger, coastal settlements. 

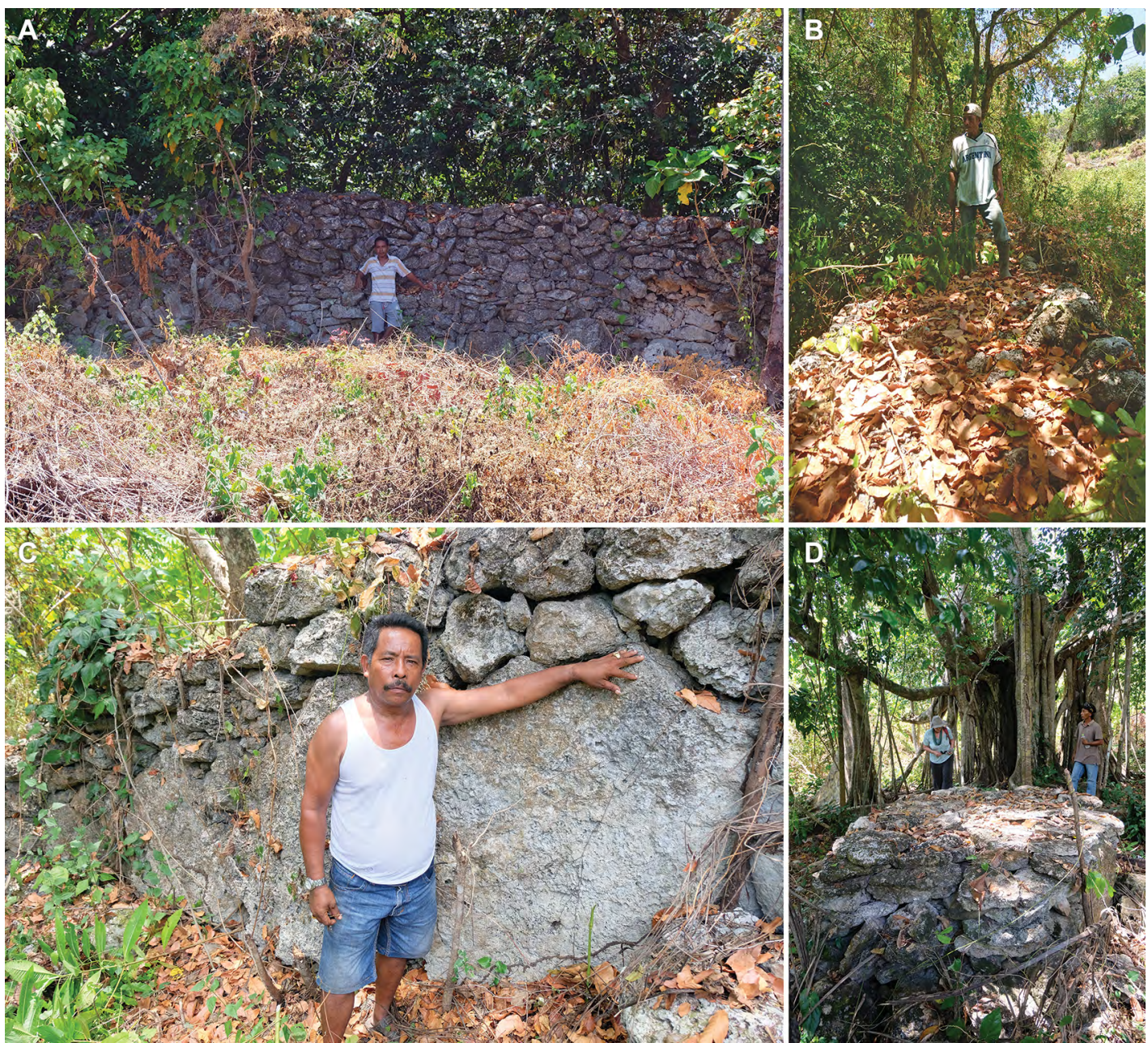

Figure 11.24. Kukeweble Negeri Lama (\#61) showing the high (A), thick (B) surrounding wall constructed of a mix of medium to very large stones $(C)$. In the centre of the Negeri Lama was a circular stone altar under the shade of a Banyan tree (D).

Source: Photos A, B and D courtesy of Shimona Kealy; photo C courtesy of Lucas Wattimena.

The largest, most complete Negeri Lama observed on Babar Besar was the Kukeweble Negeri Lama (\#61), covering an area of over 1 ha near the village of Tutuwawan. Located close to the coast on the lowest terrace, Kukeweble represents the final traditional stone-walled settlement of the Tutuwawan ancestors in their move from the mountains to the coast (Figure 11.24).

The three other Negeri Lama visited in east Babar Besar were all further inland and at higher altitude than Kukeweble. Wulua Negeri Lama (\#48) is particularly significant for its position directly on the edge of the first major terrace above the coast. The narrow stone-lined gateway to this ancestral village, located atop a c. $50 \mathrm{~m}$ steep cliff with surrounding stone walls on all other sides, would have made this Negeri Lama particularly secure against enemy forces (Figure 11.25). Wulyeni Negeri Lama (\#56) is also located on the first terrace above the coast. While the smallest of the Negeri Lamas in the area, it has an abundance of earthenware pottery and stone artefacts on the surface, suggesting good potential for in situ archaeological deposit (Figure 11.26). 


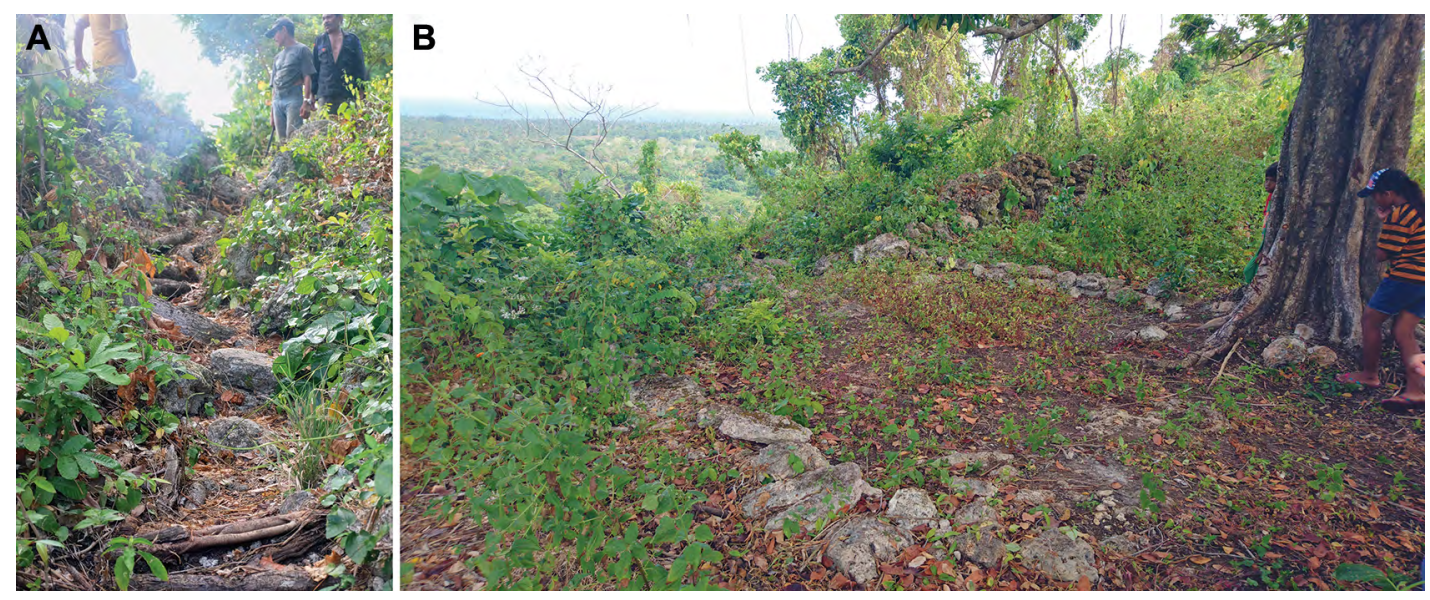

Figure 11.25. Wulua Negeri Lama (\#48) showing narrow stone staircase leading to the gateway on the cliff edge $(A)$, and stone arrangements surrounding the gateway with its commanding view over the plain below (B).

Source: Photos courtesy of Shimona Kealy.
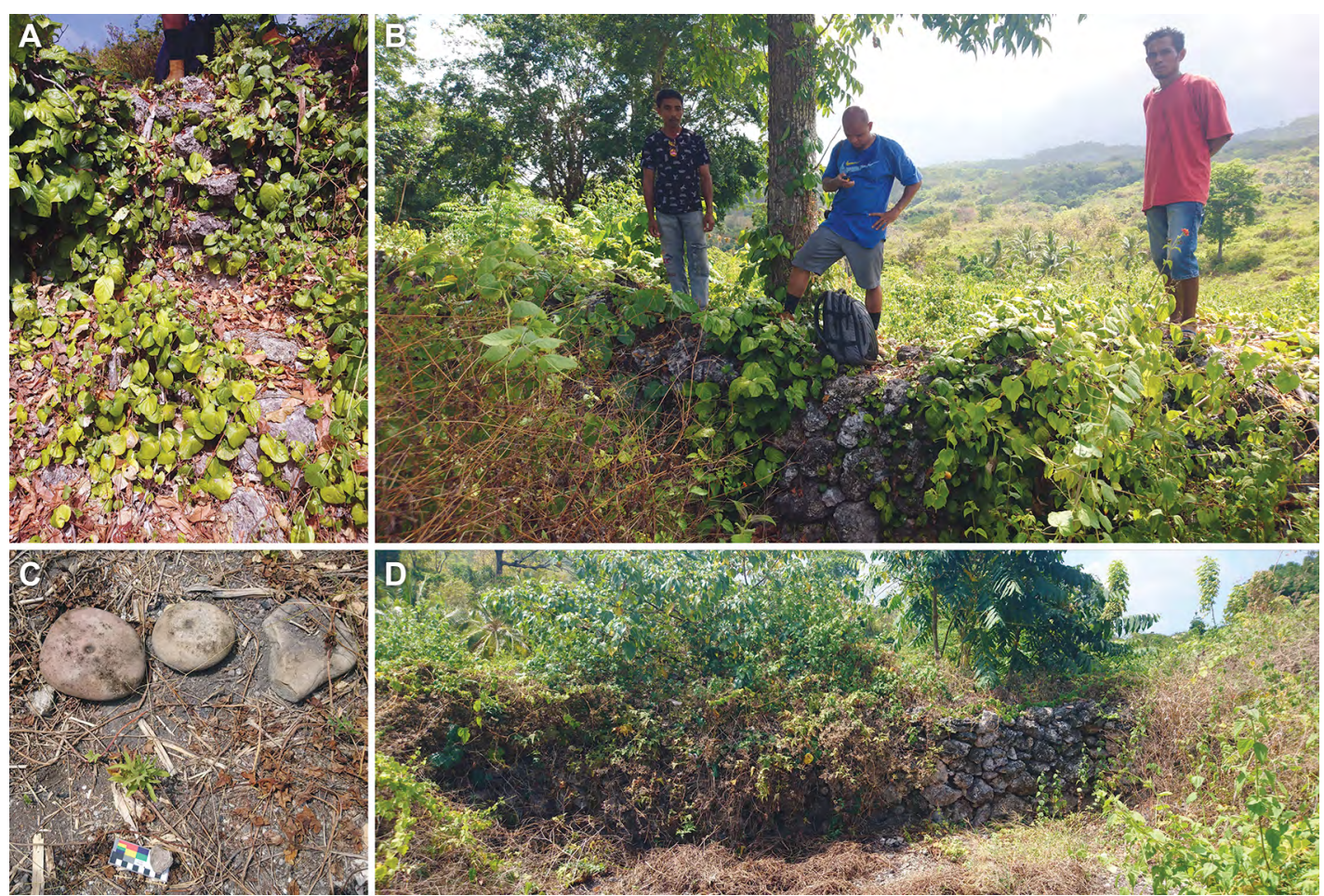

Figure 11.26. Wulyeni Negeri Lama (\#56) showing stone stair for entrance (A), circular stone wall (B and D), and stone tools located inside (C).

Source: Photos courtesy of Shimona Kealy.

The most inland fortified Negeri Lama visited was Ilkeoi (\#51), and is the second most inland fort of the series, according to the local Kokwari villagers. The oldest, more inland Negeri Lama were not visited on this survey due to their remote forested locations, time constraints and the focus of the survey being more concerned with cave and rock-shelter deposits preserving human occupation records prior to the Negeri Lama period (pre-AD 1300). 
Remote survey using satellite imagery (Google Earth Pro V 7.3.2.5491 2018b) was attempted for Babar Besar and Wetang to identify additional fortifications, as conducted on Kisar. However, these islands are more thickly forested than Kisar and the dense vegetation cover makes identification of fortifications from space virtually impossible.

Table 11.4. Summary data on fortified sites in the Babar Island group. Numbers follow full list of survey localities from Kealy et al. (2018: Table 1).

\begin{tabular}{|c|c|c|c|c|c|}
\hline${ }^{1}$ No. & Subdistrict & Site name & Location & Description & Findings \\
\hline 6 & $\begin{array}{l}\text { Wetang, Nusiata } \\
\text { Village }\end{array}$ & $\begin{array}{l}\text { Negeri Lama- } \\
\text { Nusiata }\end{array}$ & $\begin{array}{l}\text { Lat. }-07.853^{\circ} \\
\text { Long. } 129.525^{\circ} \\
{ }^{2} \text { Alt. } 111 \mathrm{~m}\end{array}$ & $\begin{array}{l}\text { Possible Negeri } \\
\text { Lama on hilltop }\end{array}$ & $\begin{array}{l}\text { Possible remains of stone structure } \\
\text { from Negeri Lama. }\end{array}$ \\
\hline 27 & $\begin{array}{l}\text { Babar Barat, } \\
\text { Manuwuy Village }\end{array}$ & $\begin{array}{l}\text { Hutmiey Negeri } \\
\text { Lama }\end{array}$ & $\begin{array}{l}\text { Lat. }-07.791^{\circ} \\
\text { Long. } 129.693^{\circ} \\
\text { Alt. } 11 \mathrm{~m}\end{array}$ & $\begin{array}{l}\text { Negeri Lama on } \\
\text { headland }\end{array}$ & $\begin{array}{l}\text { Stone wall remains indicating } \\
\text { Negeri Lama location. }\end{array}$ \\
\hline 48 & $\begin{array}{l}\text { Babar Timur, } \\
\text { Kokwari Village }\end{array}$ & $\begin{array}{l}\text { Wulua Negeri } \\
\text { Lama }\end{array}$ & $\begin{array}{l}\text { Lat. }-07.916^{\circ} \\
\text { Long. } 129.851^{\circ} \\
\text { Alt. } 47 \mathrm{~m}\end{array}$ & $\begin{array}{l}\text { Negeri Lama on } \\
\text { cliff edge }\end{array}$ & $\begin{array}{l}\text { Stone wall and gateway remains } \\
\text { of Negeri Lama. }\end{array}$ \\
\hline 51 & $\begin{array}{l}\text { Babar Timur, } \\
\text { Kokwari Village }\end{array}$ & $\begin{array}{l}\text { Ilkeoi Negeri } \\
\text { Lama }\end{array}$ & $\begin{array}{l}\text { Lat. }-07.918^{\circ} \\
\text { Long. } 129.850^{\circ} \\
\text { Alt. } 67 \mathrm{~m} \\
\end{array}$ & $\begin{array}{l}\text { Negeri Lama in } \\
\text { upper terrace }\end{array}$ & $\begin{array}{l}\text { Stone wall and gateway, largely } \\
\text { overgrown. }\end{array}$ \\
\hline 58 & $\begin{array}{l}\text { Babar Timur, } \\
\text { Manuwui Village }\end{array}$ & $\begin{array}{l}\text { Wulyeni Negeri } \\
\text { Lama }\end{array}$ & $\begin{array}{l}\text { Lat. }-07.992^{\circ} \\
\text { Long. } 129.810^{\circ} \\
\text { Alt. } 46 \mathrm{~m} \\
\end{array}$ & $\begin{array}{l}\text { Circular Negeri } \\
\text { Lama with well- } \\
\text { preserved walls }\end{array}$ & $\begin{array}{l}\text { Stone wall remains of Negeri } \\
\text { Lama. Significant surface deposit } \\
\text { of pottery and grindstones. }\end{array}$ \\
\hline 61 & $\begin{array}{l}\text { Babar Timur, } \\
\text { Tutuwawan Village }\end{array}$ & $\begin{array}{l}\text { Kukeweble } \\
\text { Negeri Lama }\end{array}$ & $\begin{array}{l}\text { Lat. }-8.005^{\circ} \\
\text { Long. } 129.806^{\circ} \\
\text { Alt. } 27 \mathrm{~m}\end{array}$ & $\begin{array}{l}\text { Large Negeri Lama } \\
\text { on headland }\end{array}$ & Large, extensive walls, stone altar. \\
\hline
\end{tabular}

Notes: ${ }^{1}$ The numbers refer to the complete archaeological survey of the island, thus some numbers are missing from this sequence. ${ }^{2}$ Approximate altitude.

Source: Kealy et al. (2018: Table 1).

It is interesting to note that on Kisar, the villagers stated that they had moved their settlements either inland or to the harbour locations, while on Babar the oldest fortifications were the furthest inland, moving progressively closer to the coast with the modern villages built along the coast. This difference could, however, be a reflection of the differences in topography and infrastructure between the two islands. Kisar is much less mountainous than Babar and has also had its interior cleared and farmed far more extensively than Babar. Additionally, while Babar is currently experiencing a boom in infrastructure construction with an airport and connecting highway to the central harbour, Kisar has had this infrastructure (and more) in place for some time. Thus, access to Kisar's centre is possible by motorised vehicles on well-built roads while the interior of Babar is only accessible on pony back via small trails.

\section{Discussion and conclusion}

The survey work undertaken to date indicates that fortification was a widespread phenomenon and was probably a feature of all occupied islands across the eastern Wallacean archipelago during the period c. AD 1400-1880. While no excavation has been undertaken in most of the fortified settlements recorded in this chapter, both the oral history and the cultural material seen on the surface inside the settlements are all consistent with their occupation within this timeframe. In some instances, oral history indicates that these settlements continued to be occupied into the early 1900s or later. For instance, in the Lautem area of Timor-Leste, oral accounts tell of ancestors fleeing their fortified settlements only when they were set on fire under instructions 
from Portuguese administrators who wanted to relocate the occupants to the coast. Fragments of tradeware, embedded in the soil inside walls and near old graves, includes Portuguese and other European ceramics dating to the mid-twentieth century. On Babar, the oral histories we collected recalled pacification and movement to the coast only in 1917.

The results of the archaeological reconnaissance reported herein indicate that the phenomenon of fort building was more geographically widespread, extending at least as far west as Sumba Island in the East Nusa Tenggara group (Figure 11.1). Schapper (Chapter 10, this volume) provides historical descriptions and photographs of fortified settlements in southern Maluku, encompassing the islands from Kisar in the west to the Kei group in the east. Looking at the historic sources for the area east of Maluku it is clear that similar defensive settlements were built as far east as Papua. Merton (1910) reported that fortifications at Ujir in the Aru Islands were already abandoned when he visited the islands in 1908 (Schapper this volume; Veth et al. 2005). Van der Crab (1864:52) reported fortified settlements built of limestone on the southern shore of the Berau Gulf, which contained deserted houses. Röder (1939-40:7) also reported fortified settlements throughout the MacCluer Gulf area of Papua. He believed that people fortified their villages due to the incursion or influence of Seramese migrants from the west and they were used as defence against firing weapons (Röder 1939-40:10). In writing of one fort in the Fu-um area of Arguni, he stated that the locals,

have for many centuries been in close contact with the population of the Isle of Ceram in the Moluccas, who sold them guns and gunpowder and probably taught them the art of building fortifications. (Röder 1939-40:10)

One of his illustrations shows an enormous roughly oval-shaped fortification with high, thick walls on the most elevated point of the mountain, which he described as already overgrown and abandoned at the time of his visit (Röder 1939-40:7). Writing in 1939, he stated that the people 'left their former fortified village and settled near the coast in Arguni some thirty years ago'. So, as with many of islands of Nusa Tenggara and Maluku discussed above, settlement along the coast apparently did not occur until the early twentieth century.

The phenomenon of fortification across eastern Wallacea has been under-documented, but remains a heritage narrative of significant historical and cultural importance worthy of further archaeological research. The data in this chapter serve as a guide and starting point for a comprehensive program of research and heritage assessment of fortified settlements, as and when appropriate funding is secured.

\section{References}

Chou, C.-Y. 2013. The evolution of natural and social landscapes in late prehistoric East Timor: A historical ecology perspective. In S. Chiu and C.-H. Tsang (eds), Archaeology and sustainability, pp. 215-235. Center for Archaeological Studies, Research Center of Humanities and Social Sciences, Academica Sinica, Taipei.

Dibley, T. 1999. Tragedy in Sumba: Why neighbours hacked each other to death in a remote part of Indonesia. Inside Indonesia 58(April-June):1-6. www.insideindonesia.org/tragedy-in-sumba. Accessed 15 February 2020.

European Space Agency 2019. Sentinels Scientific Data Hub. scihub.copernicus.eu. Accessed on 19 August 2019 and 2 March 2020.

Fitzpatrick, D., A. McWilliam and S. Barnes 2012. Property and social resilience in times of conflict: Land, custom and law in East Timor. Ashgate Press, Farnham. 
Google Earth Pro V 7.3.2.5491. 2018a. Kisar Island. 84'S $127^{\circ} 11^{\prime}$ E. C2018 AfriGIS (Pty) Ltd. Data SIO, NOAA, U.S. Navy, NGA, GEBCO. Image (C2018 DigitalGlobe.

Google Earth Pro V 7.3.2.5491. 2018b. Babar and Wetang Islands. 754'S 12943'E. Image (C2018 TerraMetrics. C2018 AfriGIS (Pty) Ltd. Data SIO, NOAA, U.S. Navy, NGA, GEBCO. Image (C)2018 DigitalGlobe.

Kealy, S., L. Wattimena and S. O'Connor 2018. A geological and spatial approach to prehistoric archaeological surveys on small islands: Case studies from Maluku Barat Daya, Indonesia. Kapata Arkeologi 14(1):1-14. doi.org/10.24832/kapata.v13i2.458.

Lape, P.V. 2006. Chronology of fortified settlements in East Timor. Journal of Island and Coastal Archaeology 1(2):285-297. doi.org/10.1080/15564890600939409.

Lieberman V. 2009. Strange parallels: Southeast Asia in context 830-1830. Volume 2. Cambridge University Press, Cambridge.

McWilliam, A., D. Bulbeck, S. Brockwell and S. O'Connor 2012. The cultural legacy of Makassar stone in East Timor. The Asia Pacific Journal of Anthropology 13(3):262-279. doi.org/10.1080/14442213. 2012.674054 .

McWilliam, A. 2007a. Harbouring traditions in East Timor: Marginality in a lowland entrepôt. Modern Asian Studies 41(6):1113-1143. doi.org/10.1017/s0026749x07002843.

McWilliam, A. 2007b. Customary claims and the public interest: On Fataluku resource entitlements in Lautem. In D. Kingsbury and M. Leach (eds), East Timor: Beyond independence, pp. 168-175. Monash Asia Institute Press, Melbourne.

Merton, H. 1910. Ergebnisse einer Zoologischen Forschungsreise in den Südöstlichen Molukken (Aruund Kei-Inseln) im Auftrag der Senckenbergischen Naturforschenden Gesellschaft. Senckenbergische Naturforschende Gesellschaft, Frankfurt am Main.

O'Connor, S., S. Pannell and S. Brockwell 2011. Whose culture and heritage for whom? The limits of national public good protected area models in East Timor. In J.N. Miksic, G.Y. Goh and S. O'Connor (eds), Rethinking cultural resource management in Southeast Asia: Preservation, development, and neglect, pp. 39-66. Anthem Press, London. doi.org/10.7135/upo9781843313588.004.

O'Connor, S., A. McWilliam, J.N. Fenner and S. Brockwell 2012. Examining the origin of fortifications in East Timor: Social and environmental factors. The Journal of Island and Coastal Archaeology 7(2):200-218. doi.org/10.1080/15564894.2011.619245.

O’Connor, S., Mahirta, D. Tanudirjo, M. Ririmasse, M. Husni, S. Kealy, S. Hawkins and Alifah 2018. Ideology, ritual performance and its manifestations in the rock art of Timor-Leste and Kisar Island, Island South East Asia. Cambridge Archaeological Journal 28(2):225-241. doi.org/10.1017/ s0959774317000816.

Pannell, S. 2006. Welcome to the Hotel Tutuala: Fataluku accounts of going places in an immobile world. The Asia Pacific Journal of Anthropology 7(3):203-219. doi.org/10.1080/14442210600965158.

Röder, J. 1939-40. Kota’s in West Nieuw-Guinea (Mac Cluer-Golf). In W.A. Hovenkamp (ed.), Tijdschrift Nieuw Guinea. Volume 4, Issue 1-6. The Nieuw-Guinea Comite en Den Nieuw-Guinea Studiekring.

Sahlins, M. 1985. Islands of history. University of Chicago Press, Chicago.

van der Crab, P. 1864. Reis naar de Zuidwestkust van Nieuw-Guinea de Goram-en Ceram-Laut Eilanden en Oostelijk Ceram. In B. van Kunsten and A. Wetenschappen (eds), Tijdschrift voor Indische Taal-, Land-, en Volkenkunde. Volume 13, pp. 531-556. 
Veth, P., S. O’Connor, M. Spriggs, W. Nayati, A. Jatmiko and H. Mohammad 2005. The Ujir site: An early historic maritime settlement in northwestern Aru. In S. O'Connor, M. Spriggs and P. Veth (eds), The archaeology of the Aru Islands, Eastern Indonesia, pp. 85-93. Terra Australis 22. ANU Press, Canberra. doi.org/10.22459/ta22.2007.05.

Viegas, S. and R. Feijó 2019. Funerary posts and Christian crosses: Fataluku cohabitations with Christian missionaries after World War II. In R. Roque and E.G. Traube (eds), Crossing histories and ethnographies: Anthropology and the colonial archive in East Timor, pp. 107-202. Berghahn Press, New York and London.

Wessel, P. and W.H F. Smith 1996. A global self-consistent, hierarchical, high-resolution shoreline database. Journal of Geophysical Research 101:8741-8743. doi.org/10.1029/96jb00104. 
This text is taken from Forts and Fortification in Wallacea: Archaeological and Ethnohistoric Investigations, edited by Sue O'Connor, Andrew McWilliam and Sally Brockwell, published 2020 by ANU Press, The Australian National University, Canberra, Australia.

doi.org/10.22459/TA53.2020.11 